\title{
The microbiota-gut-brain interaction in regulating host metabolic adaptation to cold in male Brandt's voles (Lasiopodomys brandtii)
}

\author{
Ting-Bei Bo ${ }^{1,2} \cdot$ Xue-Ying Zhang $\mathbb{1}^{1,2} \cdot$ Jing Wen ${ }^{1,2} \cdot$ Ke Deng ${ }^{1,3} \cdot$ Xiao-Wei Qin ${ }^{1} \cdot$ De-Hua Wang ${ }^{1,2}$
}

Received: 12 March 2019 / Revised: 20 July 2019 / Accepted: 24 July 2019 / Published online: 27 August 2019

(c) The Author(s), under exclusive licence to International Society for Microbial Ecology 2019

\begin{abstract}
Gut microbiota play a critical role in orchestrating metabolic homeostasis of the host. However, the crosstalk between host and microbial symbionts in small mammals are rarely illustrated. We used male Brandt's voles (Lasiopodomys brandtii) to test the hypothesis that gut microbiota and host neurotransmitters, such as norepinephrine (NE), interact to regulate energetics and thermogenesis during cold acclimation. We found that increases in food intake and thermogenesis were associated with increased monoamine neurotransmitters, ghrelin, short-chain fatty acids, and altered cecal microbiota during cold acclimation. Further, our pair-fed study showed that cold temperature can alter the cecal microbiota independently of overfeeding. Using cecal microbiota transplant along with $\beta 3$-adrenoceptor antagonism and PKA inhibition, we confirmed that transplant of coldacclimated microbiota increased thermogenesis through activation of cAMP-PKA-pCREB signaling. In addition, NE manipulation induced a long-term alteration in gut microbiota structure. These data demonstrate that gut microbiota-NE crosstalk via cAMP signaling regulates energetics and thermogenesis during cold acclimation in male Brandt's voles.
\end{abstract}

\section{Introduction}

Small mammals have evolved multiple physiological and behavioral strategies to adapt to seasonal variations in air temperature. For example, when coping with cold winters, non-hibernating small mammals increase food intake, metabolic rate, and nonshivering thermogenesis (NST) to maintain metabolic and thermal homeostasis [1-3]. Among

These authors contributed equally: Ting-Bei Bo, Xue-Ying Zhang

Supplementary information The online version of this article (https:// doi.org/10.1038/s41396-019-0492-y) contains supplementary material, which is available to authorized users.

Xue-Ying Zhang

zhangxy@ioz.ac.cn

$\triangle$ De-Hua Wang

wangdh@ioz.ac.cn

1 State Key Laboratory of Integrated Management of Pest Insects and Rodents, Institute of Zoology, Chinese Academy of Sciences, 100101 Beijing, China

2 University of Chinese Academy of Sciences, 100049 Beijing, China

3 Chengdu Institute of Biology, Chinese Academy of Sciences, 610041 Chengdu, Sichuan, China the factors which control the thermogenic process in brown adipose tissue (BAT), norepinephrine (NE) released from sympathetic fibers is one of the most important neurotransmitters used to stimulate the maximum NST in small mammals $[4,5]$. Cold acclimation leads to increased $\mathrm{NE}$ turnover in BAT, primarily through $\beta 3$-adrenergic receptors, which then initiate triglyceride breakdown into fatty acids in brown adipocytes and activates the intracellular cAMP-PKA-pCREB signaling pathway. This leads to further increased expression of uncoupling protein 1 (UCP1), a unique thermogenic protein localized in the mitochondrial inner membrane of BAT $[4,6]$. Activation of $\mathrm{NE}$ can be detected by measuring tyrosine hydroxylase $(\mathrm{TH})$, a rate-limiting enzyme in catecholamine biosynthesis [7]. Along with NE stimulation, cold-induced adaptive thermogenesis and overfeeding are also activated through increased secretion of thyroid hormones and ghrelin [8-10]. Metabolic and thermal homeostasis is traditionally thought to be regulated by the hypothalamic circuits and endocrine systems in the body [11, 12].

Increasing evidence indicates that gut microbiota play a critical role in orchestrating metabolic homeostasis of the host. Many features, such as genetics, diet, immune status, gut environment, and temperature influence gut microbial diversity [13-15], thus making gut microbiota an integral component of host energy balance [16, 17]. Resident gut 
microbiota influence host nutrition and energetics both by enhancing energy extraction from food and promoting energy storage [18, 19]. For herbivorous animals, gut microbiota can degrade fiber in food to produce short-chain fatty acids (SCFAs) and vitamins. SCFAs can directly interact with the enteric nervous system (ENS), providing a causal link between environment-induced alterations in gut microbiota and the physiology and behavior of the host $[20,21]$. It has been reported that common SCFAs, such as acetate, propionate, and butyrate, can stimulate gut hormone secretions, reduce appetite, and enhance both fatty acid oxidation and BAT thermogenesis [20, 22, 23]. Recent data indicate that gut microbiota also regulate 5hydroxytryptamine (5-HT) biosynthesis and ENS maturation, ultimately influencing brain development and functions of the host [23-26]. This communication between the brain and gut is bidirectional-the brain also largely controls the gut.

Brandt's voles (Lasiopodomys brandtii), a nonhibernating herbivorous species, mainly inhabit the grasslands of Inner Mongolia of China, Mongolia, and the region of Beigaer in Russia. As winter lasts for over 5 months in these habitats with an average annual temperature of $0-4{ }^{\circ} \mathrm{C}$, Brandt's voles provide a natural model for studying the effects of cold acclimation [2]. Brandt's voles have evolved typical physiological responses to this cold environment, including reduced fat mass, increased food intake, increased BAT mass and increased NST [2, 27], and increased intestinal villus length to improve digestion [28]. They have also evolved huddling behavior to help survive the winter. Both cold temperature exposure and huddling behavior affect body temperature and cecal microbiota in Brandt's voles [29]. However, the crosstalk between the host and microbial symbionts in small mammals are rarely illustrated. We hypothesized that gut microbiota interact with neurotransmitters to mediate cold-induced thermogenesis. Here, we illustrate that cold temperature exposure affected gut microbiota and metabolites, and this is not due to overfeeding. We also found that cold temperatureexposed microbiota regulates $\mathrm{NE}$ release in the small intestine and BAT by activating cAMP signaling, and this contributes to winter metabolic phenotypes. Lastly, we found that host neurotransmitters (such as NE) induced long-term alterations in gut microbiota in Brandt's voles.

\section{Materials and methods}

\section{Animals}

All animals were licensed under the Animal Care and Use Committee of the Institute of Zoology at the Chinese Academy of Sciences. Subjects were offspring of Brandt's voles trapped in Inner Mongolian grasslands in May 1999 that have been raised at the Institute of Zoology, the Chinese Academy of Sciences in Beijing [2]. Breeding colonies were rejuvenated either with a wild population or with another laboratory every 3 years. Subjects were housed in groups (3-4) in plastic cages $\left(30 \times 15 \times 20 \mathrm{~cm}^{3}\right)$ with sawdust bedding after weaning and were maintained at a room temperature of $23 \pm 1{ }^{\circ} \mathrm{C}$ under a photoperiod of $16 \mathrm{~L}: 8 \mathrm{D}$. Adult male Brandt's voles (about 5 months old) had free access to water and commercial rabbit pellets (Beijing KeAo Feed Co.). All subjects were housed individually in cages at least 2 weeks before experiments.

\section{Experimental designs}

Experiment 1 was designed to test how physiological phenotypes and cecal microbiota change during cold and rewarming acclimations in Brandt's voles. Thirty-two adult male voles were randomly assigned into one of two groups (treatment and control groups, 16 voles per group). The treatment group was transferred to a cold $\left(4 \pm 1{ }^{\circ} \mathrm{C}\right)$ room for 4 weeks (C4), or first transferred to a cold room for 4 weeks and were then returned to a warm $\left(23 \pm 1^{\circ} \mathrm{C}\right.$, rewarming) room for another 4 weeks (C4W4). The control group remained at $23 \pm 1{ }^{\circ} \mathrm{C}$ for 4 (W4) or 8 weeks (W4W4). After the acclimation period, we measured body mass, food intake, UCP1 expression, monoamine transmitter levels, cecal microbiota, and microbial metabolites (SCFAs).

Experiment 2 verified whether cold temperature rather than overfeeding induced changes in cecal microbiota. Twenty-four adult male voles were divided into four groups ( $n=6$ for each group): a food-restricted (FR, 80\% of the free-fed food intake) group at room temperature (FR $23^{\circ} \mathrm{C}$ ), a free-fed control group at room temperature $\left(\operatorname{Con} 23{ }^{\circ} \mathrm{C}\right)$, a food-restricted group at a cold room temperature $\left(\mathrm{FR} 4{ }^{\circ} \mathrm{C}\right)$, and a free-fed group at a cold room temperature $\left(\mathrm{Con} 4{ }^{\circ} \mathrm{C}\right)$ named FR $23{ }^{\circ} \mathrm{C}$ and $\mathrm{Con} 23^{\circ} \mathrm{C}$, and another two groups at cold room $\left(4 \pm 1{ }^{\circ} \mathrm{C}\right.$ ) named FR $4{ }^{\circ} \mathrm{C}$ (pair-fed as Con $23{ }^{\circ} \mathrm{C}$ ) and Con $4{ }^{\circ} \mathrm{C}$ ( $n=6$ for each group). Body mass, resting metabolic rate (RMR), NST, and cecal microbiota were measured during the 4 weeks of acclimation.

Experiment 3 investigated the role of gut microbiota in mediating host metabolic phenotypes through cecal microbiota transplant (CMT). Three voles from groups $\mathrm{C} 4$ and W4 (Experiment 1) respectively were used as donors. Twenty-four adult male voles were divided into four groups: a control group (without any treatment), an antibiotic group ( $\mathrm{Ab}$, which received sterile saline by gavage after antibiotics stopped), a group that were transplanted with cold temperature-exposed microbiota after antibiotics stopped (R-C4), and a group that were transplanted with warm temperature-exposed microbiota after antibiotics 
stopped (R-W4). Ab, R-C4, and R-W4 groups were first treated with fresh composite antibiotics (containing $100 \mu \mathrm{g} /$ $\mathrm{mL}$ neomycin, $50 \mu \mathrm{g} / \mathrm{mL}$ streptomycin, and $100 \mathrm{U} / \mathrm{mL}$ penicillin; Sigma, Germany) via intragastric gavage $(200 \mu \mathrm{L})$ daily for 3 days. The cecal content $(200 \mathrm{mg})$ from the donors was suspended in sterile $0.9 \%$ saline $(2 \mathrm{~mL})$ and centrifuged to get the bacterial suspension. The bacterial suspension or sterile $0.9 \%$ saline $(200 \mu \mathrm{L})$ was then transplanted via intragastric gavage to antibiotictreated recipient voles. The recipients were housed individually at room temperature $\left(23 \pm 1{ }^{\circ} \mathrm{C}\right)$ and $16 \mathrm{~L}: 8 \mathrm{D}$ after transplant. Body mass, food intake, RMR, and NST were measured after CMT for 1 week. UCP1 expression in BAT and the levels of monoamine transmitters were measured. DNA from cecal contents were extracted for 16S rRNA sequencing.

Experiment 4 examined the role of the cAMP-PKA pathway in mediating host metabolic phenotypes after CMT. Voles from groups C4 and W4 (Experiment 1) were used as donors. Twenty-four adult male voles were treated with antibiotics and then transplanted with cold temperature-exposed microbiota (R-C4) via intragastric gavage. Three days later, subjects were divided into three groups ( $n=8$ per group) that were intraperitoneally injected with either $0.1 \mathrm{~mL}$ sterile $0.9 \%$ saline, H89 (a PKA inhibitor, $100 \mu \mathrm{g} / \mathrm{kg}$ body mass; Abcam), or SR 59230A (a $\beta 3$-adrenoceptor antagonist, $1 \mathrm{mg} / \mathrm{kg}$ body mass; SigmaAldrich). An additional set of voles $(n=8)$ were transplanted with warm temperature-exposed microbiota (R-W4) and received $0.1 \mathrm{~mL}$ sterile $0.9 \%$ saline. Antibiotic treatment and CMT were the same as those in experiment 3. After 2 days of injection, body mass, NST, UCP1 expression in BAT, and cAMP-PKA and p-CREB/CREB in BAT and the small intestine were measured. All subjects were kept at $23 \pm 1{ }^{\circ} \mathrm{C}$ and $16 \mathrm{~L}: 8 \mathrm{D}$.

Experiment 5 examined the role of NE in regulating gut microbiota and metabolic phenotypes. Thirty-two adult male voles were divided into four groups that received either NE manipulation for 7 days (NE), NE manipulation and then restored for 7 days (NE-R7), NE manipulation and then restored for 21 days (NE-R21), or no NE manipulation (control) for 4 weeks ( $n=8$ per group). Voles received either $0.9 \%$ saline or $\mathrm{NE}(\mathrm{mg} / \mathrm{kg})=2.53 \mathrm{~W}^{-0.4}$ (dissolved in $0.9 \%$ saline) via intraperitoneal injection [30] twice per day for 1 week. Body mass, food intake, body temperature, RMR, NST, UCP1 expression in BAT, monoamine neurotransmitter levels, and fecal microbiota were measured. Subjects were kept at $23 \pm 1{ }^{\circ} \mathrm{C}$ and $16 \mathrm{~L}: 8 \mathrm{D}$.

\section{Measurement of body mass and food intake}

Body mass and food intake were measured at 9:00 a.m. using an electronic balance (Sartorius Model BL 1500, $\pm 0.1 \mathrm{~g}$ ). Food intake (g) was calculated by subtracting uneaten food weight from initial food weight.

\section{Measurement of RMR and NST}

RMR was measured at $30^{\circ} \mathrm{C}$ in $2.7 \mathrm{~L}$ transparent metabolic chambers (Type I for mice) [29]. The air flow rate was $0.8 \mathrm{~L} / \mathrm{min}$. RMR was measured through oxygen consumption by using an open-circuit respirometry system (TSE labmaster, Germany) for $3 \mathrm{~h}$. RMR was calculated by averaging a minimum of three consecutive, stable readings of oxygen consumption after $1 \mathrm{~h}$ of acclimation.

NST was determined at $25^{\circ} \mathrm{C}$ on the day after RMR measurement. Subjects were injected subcutaneously with NE (Shanghai Harvest Pharmaceutical Co. Ltd). The dosage of NE was calculated using a formula $(\mathrm{NE}(\mathrm{mg} / \mathrm{kg})=2.53$ $\mathrm{W}^{-0.4}$ ) for Brandt's voles $[29,30]$. NST was estimated from the three highest consecutive, stable readings of oxygen consumption 15-20 min post-injection during the one-hour measurement.

\section{Measurement of T3, T4, and ghrelin in serum}

Serum T3 and T4 concentrations were quantified via radioimmunoassay using an RIA kit (China Institute of Atomic Energy, Beijing, China) according to the instructions. The minimum detectable concentration of the assay (sensitivity) when using a $50 \mu \mathrm{L}$ sample was $0.25 \mathrm{ng} / \mathrm{mL}$ for $\mathrm{T} 3$ and $3.96 \mathrm{ng} / \mathrm{mL}$ for T4. Intra- and inter-assay coefficients of variation were $2.4 \%$ and $8.8 \%$ for $\mathrm{T} 3$, and $4.3 \%$ and $7.6 \%$ for $\mathrm{T} 4$, respectively.

Serum ghrelin (a des-acylated form) concentrations were measured using a Ghrelin EIA kit (Catalog No. EK-031-31, Phoenix Pharmaceuticals, USA). The minimum detected concentration of the kit was $0.13 \mathrm{ng} / \mathrm{mL}$. The intra- and inter-assay variations for ghrelin EIA were $10 \%$ and $15 \%$, respectively.

\section{Measurement of UCP1 by immunohistochemistry}

Interscapular WAT was fixed in Bouin's fluid overnight with paraffin embedded, cut in 5- $\mu \mathrm{m}$-thick sections, and stained with hematoxylin-eosin (H\&E) using standard techniques, and was incubated in a rabbit anti-UCP1 polyclonal primary antibody (1:5000; Merck Millipore). Immunoreactivity was identified using biotinylated goat anti-mice immune globulin G (1:3000; Jackson ImmunoResearch, USA). Sections were then incubated with ABC reagents (PK-6100; Vector Labs, USA) for $60 \mathrm{~min}$. Immunoreactive sites were subsequently identified by 3,3 diaminobenzidine (DAB substrate kit; Vector Labs, USA), and tissue sections were photographed using a Nikon optical microscope (Nikon H600L). 


\section{Measurement of UCP1, TH, CAMP, PKA, and pCREB by western blot}

Jejunum segments $(1.5 \mathrm{~cm}$, whole thickness of tissue), BAT $(\sim 0.1 \mathrm{~g})$ and WAT $(\sim 0.1 \mathrm{~g})$ were homogenized in RIPA buffer and cleared by centrifugation, according to the standard techniques [2, 3, 27]. Western blots of wholetissue lysates were probed with primary antibodies against UCP1 (ab155117; Abcam), TH (AB152; Merck Millipore), Anti-cAMP Protein Kinase Catalytic subunit antibody (ab26322; Abcam), Phospho-CREB (pCREB, 9198; Cell signaling), CREB (9197; Cell signaling), FFAR2/GPR43 (ABC299; Merck Millipore), and GAPDH (A01020; Abbkine). The secondary antibody used was either peroxidaseconjugated goat anti-rabbit IgG (111-035-003; Jackson), or peroxidase-conjugated goat anti-mice IgG (115-035-003; Jackson). Protein markers (20351ES76; Shanghai Yisheng, China) were added on both sides of each gel to verify bands. The PVDF membranes were detected by enhanced chemoluminescence (Beyotime, China). Bands were analyzed using Image $\mathrm{Lab}^{\mathrm{TM}}$ Software (Bio-Rab Laboratories), were normalized to GAPDH and expressed as relative units (RU).

\section{Measurement of monoamine transmitters}

Dopamine (DA), 5-HT, and their metabolites (3,4-dihydroxyphenylacetic acid (DOPAC), 5-hydroxyindoleacetic acid (5-HIAA)), along with NE levels in BAT, small intestine, and hypothalamus were measured via reversed phase UltiMate High Performance Liquid Chromatography with electrochemical detection (UHPLC-ECD, DIONEX UltiMate 3000, RS Pump). The internal standard was 3,4dihydroxybelzyamine. Average weights of tissue sampled were as follows: hypothalamus $\sim 0.015 \mathrm{~g}$, jejunum segments $\sim 0.1 \mathrm{~g}$, and BAT $\sim 0.06 \mathrm{~g}$. Samples were homogenized in $0.1 \mathrm{M}$ cold perchloric acid, went under ultrasonic oscillation for $10 \mathrm{~s}$, and were centrifuged at $13,000 \mathrm{RPM}$ at $4{ }^{\circ} \mathrm{C}$ for $30 \mathrm{~min}$. Supernatant was filtered using a $0.2 \mathrm{~mm}$ nylon filter. Aliquots of $30 \mu \mathrm{L}$ were manually injected using a Hamilton syringe. The mobile phase $(\mathrm{pH}=4.0)$ consisted of $90 \mathrm{mM} \mathrm{NaH} \mathrm{PO}_{4} 2 \mathrm{H}_{2} \mathrm{O}, 50 \mathrm{mM}$ citric acid monohydrate, $1.7 \mathrm{mM}$ alkylsulfonate (SAS), $50 \mu \mathrm{M}$ EDTA, $2 \mathrm{mM} \mathrm{NaCl}$, and $8 \%$ Acetonitrile. The detector (Thermo Scientific Ultimate 3000 RS Electrochemical Detector with 6041RS Ultra Amperometric Cell (6070.3000), highly efficient glassy carbon electrode (6070.3200), 6041RS sensor, and Pd reference electrode) was set at $150 \mathrm{mV}$, and the column (Acclaim $^{\text {TM }} 120$, C18 $3 \mathrm{~m} 120 \AA, 2.1 \times 150 \mathrm{~mm}$ ) was placed at $8{ }^{\circ} \mathrm{C}$. Compounds were identified by comparing their retention times with those of authentic reference compounds. The level of each monoamine was calculated by the internal standard method using Chromeleon 7 software (ThermoFisher Scientific, MA, USA).
Quantitative calculation:

(1) The samples were quantified according to the correction factor+internal standard method and were converted into $\mathrm{ng} / \mathrm{mg}$ of wet tissue.

Correction factor $(f)=$ (peak area of internal standard substance/concentration of internal standard substance)/(peak area of standard/concentration of standard).

(2) Content $($ sample $)=f \times$ peak area of sample $/($ peak area of internal standard substance/concentration of internal standard substance)\}.

(3) The amount of material was measured by $W(\mathrm{ng})=$ Content (sample) $\times V$ (volume of liquid to be measured). Monoamine and metabolite levels are expressed as $\mu \mathrm{g} / \mathrm{g}$ of wet tissue. Ratio of metabolite(s)/ amine was calculated as an index of rate of metabolism; DOPAC/DA indicated dopamine turnover rate and 5-HIAA/5-HT indicated 5-HT turnover rate.

\section{$16 S$ rRNA gene profiling}

After animals were sacrificed by $\mathrm{CO}_{2}$ overdose between 9:00 and 11:00 a.m., fresh gut contents were collected from the cecum and colon, were immediately frozen, and were stored in $-80^{\circ} \mathrm{C}$. DNA from cecal or fecal contents was extracted by $2 \times$ cetyltrimethyl ammonium bromide $(2 \times$ $\mathrm{CTAB}$ ) and phenol chloroform mixture (phenol:chloroform: isoamyl alcohol $=25: 24: 1$ ), and was then isolated by a spin column using SanPrep Column DNA Gel Extraction Kit (Sangon Biotec, B518131-0100) [29]. Universal primers were used for PCR amplification of the V3-V4 hypervariable regions of $16 \mathrm{~S}$ rRNA genes $[29,31]$ and contained Illumina 3'adaptor sequences as well as a 12-bp barcode. Sequencing was done on an Illumina HiSeq 2500. Raw sequencing reads were de-noised, filtered according to barcode and primer sequences (Forward primer-341F, CCTACGGGNGGCWGCAG; Reverse primer-805R, GACTACHVGGGTATCTAATCC), and classified with the Quantitative Insights Into Microbial Ecology (QIIME, version 1.9.1) software suite.

Chimeric sequences were removed using usearch61 with de novo models. The open-reference Operation Taxonomic Unit (OTU) picking strategies in QIIME software suite were used to identify OTU clustering algorithm. In an openreference OTU picking process, reads were clustered against the 2013 Greengenes (13_8 release) ribosomal database's $97 \%$ reference data set (ftp://greengenes. microbio.me/greengenes_release/gg_13_5/gg_13_8_otus.ta r.gz) and any reads which did not hit the reference sequence collection were subsequently clustered into de novo OTUs 
at $97 \%$ similarity with UCLUST. Taxonomy was assigned to the OTUs using the RDP classifier within QIIME and Greengenes reference data set. Rarefaction and rank abundance curves were calculated from OTU tables using alpha diversity and rank abundance scripts within the QIIME pipeline. The hierarchical clustering based on population profiles of most common and abundant taxa was performed using UPGMA clustering (Unweighted Pair Group Method with Arithmetic mean, also known as average linkage) on the distance matrix of OTU abundance. This resulted in a Newick formatted tree, which was obtained utilizing the QIIME package. Nonmetric multidimensional scaling (NMDS) based on unweighted and weighted UniFrac distance metrics was used to visualize the structure of microbial community ( $\beta$ diversity). The $\beta$ diversity was also indicated by the principal coordinate analysis (PCoA) based on unweighted and weighted UniFrac distance metrics with OTUs for each sample in experiment 2.

\section{Measurement of SCFAs}

We measured six SCFAs: acetic acid, propionic acid, butyric acid, isobutyric acid, valeric acid, and isovaleric acid from cecal contents. SCFAs were measured via high performance gas chromatography (GC) (Agilent 7890A; Agilent Technologies, Germany) with a GC autosampler and an FID system [29]. Separations were performed in a $30 \mathrm{~m} \times 0.25 \mathrm{~mm} \times 0.25 \mu \mathrm{m} \quad$ DB-WAX column (Agilent Technologies). In total, $99.998 \%$ hydrogen was used as carrier gas at a flow-rate of $1.0 \mathrm{~mL} / \mathrm{min}$. The system was operated at $250{ }^{\circ} \mathrm{C}$. Injections were performed in the splitless mode at $230{ }^{\circ} \mathrm{C}$, and $0.5 \mu \mathrm{L}$ for each injection. The oven temperature was programmed from $60^{\circ} \mathrm{C}(1 \mathrm{~min})$ at $5{ }^{\circ} \mathrm{C} / \mathrm{min}$ to $200{ }^{\circ} \mathrm{C}$ and then from $200{ }^{\circ} \mathrm{C}$ at $10{ }^{\circ} \mathrm{C} / \mathrm{min}$ to $230^{\circ} \mathrm{C}$. The total running time of each sample was $32 \mathrm{~min}$.

\section{Statistical analysis}

Data were analyzed using SPSS 20.0 (SPSS Inc., Chicago, IL, USA). Normality and homogeneity of variance of the data were examined using Shapiro-Wilk and Levene tests, respectively. Body mass and food intake during the acclimation were analyzed by repeated measures ANOVA or ANCOVA followed by Tukey's post hoc tests. Differences in RMR and NST between groups were analyzed by oneway or two-way ANCOVA with body mass as the covariate followed by Tukey's post hoc tests. SCFAs content, TH, UCP1, cAMP-PKA and pCREB protein expression, monoamine transmitter levels, serum hormones, and microbiota abundance were all analyzed by independent $t$ test, one-way or two-way ANOVA with Tukey's post hoc tests. Pearson correlation analyses were used to determine the relationship between metabolic parameters. Results are presented as means $\pm \mathrm{SE}$, and the level of statistical significance was set at $p<0.05$.

The significance for NMDS or PCoA analyses was detected with multivariate permutation tests using the nonparametric method "ANOSIM" (permutations =999) included in the $\mathrm{R}$ "vegan" package of the QIIMEincorporated version. The linear discriminant analysis (LDA) Effect Size (LEfSe) method was used to assess differences in microbial communities using a LDA score threshold of 2 [29]. Bootstrapping (--permutations 1000) and Pearson correlation were used to calculate the correlation between specific OTUs and physiological measurements. The level of statistical significance was set at $p<$ 0.05 (false discovery rate corrected).

\section{Results}

\section{Cold and rewarming induce alterations in metabolic phenotypes and monoamine neurotransmitters}

To test how physiological phenotypes changed from cold temperature and rewarming, we either acclimated the voles to a cold room $\left(4 \pm 1{ }^{\circ} \mathrm{C}\right)$ for 4 weeks and then transferred them back to the warm room $\left(23 \pm 1^{\circ} \mathrm{C}\right)$ for another 4 weeks, or kept in warm conditions to serve as controls (Fig. 1a). Body mass showed no differences between groups $\left(F_{1,14}=0.105, p=0.75\right.$, Fig. S1a). Food intake increased significantly in the second and fourth weeks of cold acclimation, and intake remained higher during the first two weeks of rewarming compared to controls. There were no differences in food intake during the last week of warm acclimation $\left(F_{1,14}=17.602, p=0.001\right.$, Fig. 1b). Voles kept in the cold room for 4 weeks (C4) had $25 \%$ higher UCP1 expression in BAT compared to voles in the warm room (W4) $(t=7.596, \mathrm{df}=14, p<0.01)$, and the C4W4 group did not show any differences from the W4W4 group $(t=$ $1.044, \mathrm{df}=14, p=0.314$, Fig. 1c). Based on the immunohistochemistry for the UCP1 protein marker of white adipocytes, the total UCP1-positive area proportion in the $\mathrm{C} 4$ group increased significantly compared to the $\mathrm{W} 4$ group ( $t=9.246, p<0.001)$, whereas the C4W4 group showed no differences from the W4W4 group $(t=1.679, p=0.104$, Fig. 1d). There were two types of adipocytes: UCP1positive multilocular white adipocytes (described as beige adipocytes, indicated by the white arrow) and UCP1-negative unilocular adipocytes (described as white adipocytes, indicated by the black arrow) (Fig. S1b). The des-acylated ghrelin levels in serum of the $\mathrm{C} 4$ group was higher than the W4 group $(t=2.636, p=0.02$, Fig. 1e). The $\mathrm{C} 4$ group had a higher T3/T4 ratio than the $\mathrm{W} 4$ group $(t=3.487, p=0.006$, Fig. 1f), whereas there were no differences between the C4W4 and W4W4 groups. Both T3 
a

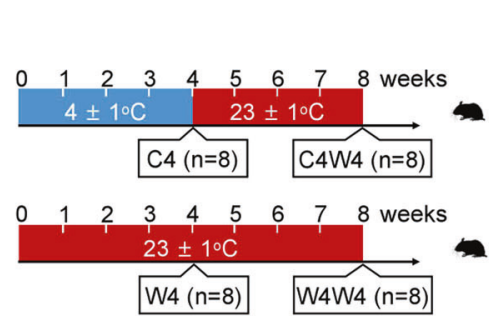

d

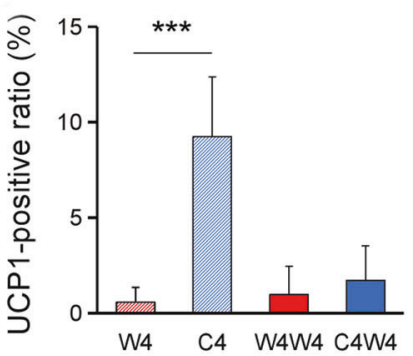

g

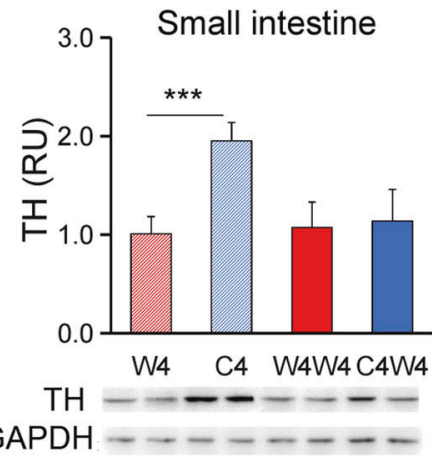

b

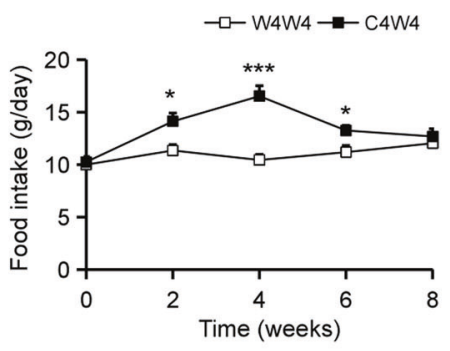

e

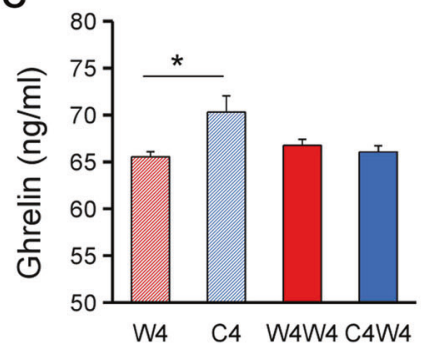

h

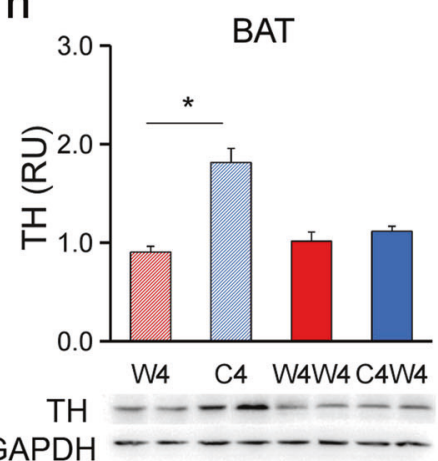

C

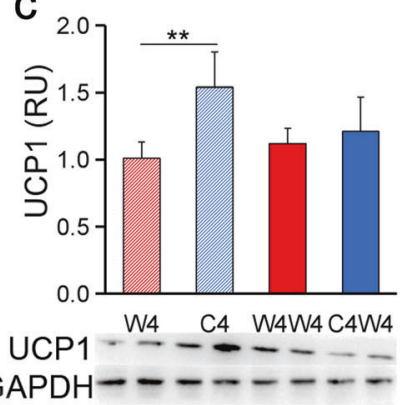

$f$

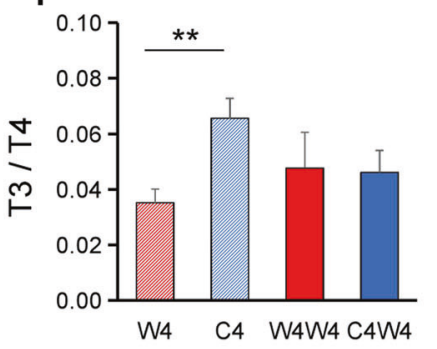

i

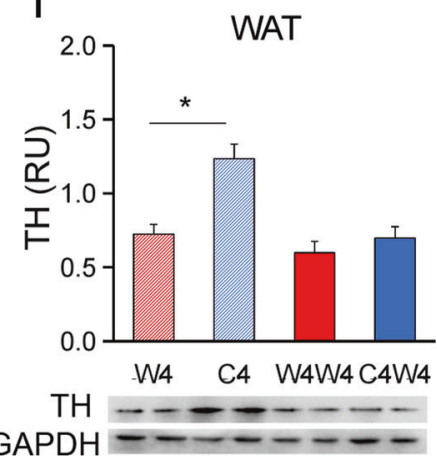

Fig. 1 Cold and rewarming induce alterations in metabolic phenotypes and monoamine neurotransmitters. a The design paradigm. b Changes of food intake with time of acclimation (repeated measures ANOVA). c Uncoupling protein 1 (UCP1) expression in interscapular brown adipose tissue (BAT) (UCP1 and others, independent $t$-test between W4 and C4, W4W4 and C4W4). d The ratio of UCP1-positive expression in white adipose tissue (WAT). e Ghrelin level in serum. f $\mathrm{T}_{3} / \mathrm{T}_{4}$ ratio in serum. $\mathrm{g}-\mathbf{i}$ Tyrosine hydrogenase $(\mathrm{TH})$ expression in small intestine, BAT and WAT. W4, warm condition for 4 weeks; C4, cold acclimation for 4 weeks; W4W4, warm condition for 8 weeks; C4W4, cold acclimation for 4 weeks and then back to warm condition for 4 weeks. Data are means $\pm \operatorname{SEM~(~} n=8$ per group). $* p<0.05$, $* * p<0.01$, and $* * * p<0.001$
$(R=0.689, p<0.001)$ and ghrelin levels $(R=0.431, p=$ $0.014)$ were positively correlated with UCP1 expression in BAT (Fig. S1c, d).

$\mathrm{TH}$ expression in the small intestine $(t=3.680 \mathrm{df}=14$, $p=0.002)$, BAT $(t=5.884, p<0.001)$, and WAT $(t=$ $4.279, p=0.001)$ increased in the $\mathrm{C} 4$ group compared to that of the W4 group, whereas there was no difference between the C4W4 and W4W4 groups (Fig. 1g-i). NE levels increased in the BAT, small intestine, and hypothalamus of the $\mathrm{C} 4$ group compared to the W4 group (Fig. S1e-g). Significant increases in both dopamine turnover rate (DOPAC/DA ratio) in the small intestine and hypothalamus (Fig. S1h-j) and 5-HT turnover rate (5HIAA/5-HT ratio) in the BAT and small intestine were observed after cold acclimation (Fig. S1k-m). These data suggest that the voles adjusted the plasticity of their metabolic physiology in response to variations in air temperature.

\section{Cold and rewarming alter gut microbiota and metabolites}

To identify how cold and rewarming exposure affects gut microbiota and bacterial metabolites, we analyzed $16 \mathrm{~S}$ rRNA gene sequencing and measured the concentration of SCFAs in cecal contents. After removing low-quality sequences, chloroplasts, chimeras, and singletons, we obtained 587,400 valid sequences. At a threshold of $97 \%$ 
sequence identity, 33,158 unique OTUs were identified in our results. The OTU-level rarefaction curve of Goods coverage across all samples reached stable values (Fig. S2a), showing that most of the cecal microbial diversity had already been captured in our study despite the possibility of detecting rare new OTUs with additional sequencing depth. The alpha diversity of vole's cecal microbiota indicated by the PD_whole tree increased in the $\mathrm{C} 4$ group compared to the $\mathrm{W} 4$ group $(t=0.520, \mathrm{df}=14$, $p=0.035$ ) (Table S1, Figs. 2a and S2b). For $\beta$ diversity, analysis based on unweighted (ANOSIM, $R=0.220, p=$ 0.001 , Fig. 2b) and weighted UniFrac distance $(R=0.090$, $p=0.039$, Fig. S2c) showed that the cecal microbiota structure of the $\mathrm{C} 4$ group was separated from the W4 group. LEfSe method with LDA score $>2$ identified the different microbial communities among groups (Fig. 2c, S2d). Firmicutes and Bacteroidetes were the most abundant phyla (91.2\% on average) in all samples (Fig. S2e), and significant differences were observed at the phylum level in Bacteroidetes and Firmicutes (Fig. 2d). The Firmicutes phylum was increased in abundance by up to $55.2 \%$ during cold exposure (compared to $45 \%$ in $\mathrm{W} 4$ ), and Bacteroidetes phylum abundance decreased to $37.8 \%$ (compared to $49.4 \%$ in W4). At the family level, the proportions of Clostridiaceae and [Odoribacteraceae] increased, whereas S24-7 decreased in $\mathrm{C} 4$ voles compared to W4 voles (Table S2). At the genus level, proportions of Odoribacter, Paraprevotella, Clostridium, [Ruminococcus], and Helicobacter in the $\mathrm{C} 4$ group were higher than the W4 group (Table S3).

The total concentration of SCFAs in the $\mathrm{C} 4$ group was significantly higher than in the W4 group $(t=4.417, \mathrm{df}=$ $12, p=0.001$, Fig. S2f). The ratio of acetic acid was $71 \%$ in $\mathrm{W} 4,67 \%$ in $\mathrm{C} 4,72 \%$ in W4W4, and $73 \%$ in C4W4. On the contrary, the ratio of butyric acid was $19 \%$ in C4 voles compared to $16 \%$ in $\mathrm{W} 4$ voles. The concentrations of acetic acid $(t=2.658, \mathrm{df}=12, p=0.021)$, propionic acid $(t=$ 2.696, $p=0.019)$, butyric acid $(t=3.392, p=0.005)$, and isobutyric acid $(t=2.732, p=0.018)$ in the $\mathrm{C} 4$ group were higher than those in the W4 group (Fig. S2g). The concentration of isovaleric acid in the $\mathrm{C} 4$ group was lower than in the W4 group $(t=2.868, p=0.014)$, and the valeric acid concentration was also lower in the $\mathrm{C} 4$ group $(t=$ 2.827, $p=0.015$ ). The concentrations of these six SCFAs were not different between the W4W4 and C4W4 groups (Fig. S2g).

The heatmap showed the correlation between specific OTUs and physiological measurements (Fig. 2e). The genera of Coprococcus, Prevotella, and Ruminococcus were correlated with ghrelin levels in serum. Lactobacillus and Ruminococcus were correlated with $\mathrm{T} 3$ levels in serum. Oscillospira and Ruminococcus were correlated with food intake. Lactobacillus, Oscillospira, Prevotella, Roseburia, and Ruminococcus were correlated with NE levels in BAT, and UCP1 expression in BAT and WAT. These data indicate that the variations in gut microbiota may be associated with the plasticity of metabolic physiology during temperature acclimation.

\section{Cold temperature rather than overfeeding in cold influences gut microbiota structure}

To verify that cold temperature, rather than overfeeding, induced changes in gut microbiota, we fed cold-exposed voles with the same amount of food as room temperature voles (pair-fed manipulation, Fig. 3a). The voles lost more body mass when they were restricted of food in both warm and cold settings $\left(F_{1,20}=38.659, p<0.001\right.$; Fig. $\left.3 b\right)$. Food restriction did not affect RMR $\left(F_{1,19}=0.130, p=0.722\right.$; Fig. 3c), but it induced a reduction in NST $\left(F_{1,19}=4.354\right.$, $p=0.051$, Fig. 3d). Voles in the cold condition had a higher $\alpha$-diversity, indicated by observed OTUs and Shannon Index, than voles in the warm condition $(p<0.05)$, but the food effect was not significant ( $p>0.05$, Figs. 3e and S3a). The profile of cecal microbiota composition indicated by NMDS and PCoA based on weighted (Fig. 3f, g) and unweighted (Fig. S3b, c). UniFrac distance, showed that cold temperature (weighted, ANOSIM, $R=0.741, p=$ 0.001 ; unweighted, ANOSIM, $R=0.338, p=0.002$ ), rather than food restriction (weighted, ANOSIM, $R=0.046, p=$ 0.776; unweighted, ANOSIM, $R=0.021, p=0.546$ ), significantly influenced cecal microbiota structure. LEfSe methods identified the different microbial communities in the four groups (Fig. S3d, e). We observed differences in OTUs abundance at phylum level in Bacteroidetes, Firmicutes, Proteobacteria, and TM7 between warm and coldexposed voles $(p<0.05$, Fig. S3f, g). At the family level, the abundance of Oxalobacteraceae, Veillonellaceas, Peptococcaceae, Mogibacteriaceae, Desulfovibrionaceae, Helicobacteraceae, Ruminococccaceae, F16, and S24-7 showed significant differences between warm and cold-exposed voles $(p<0.05$, Fig. S3h). No difference at the phylum or family levels was observed between FR $4{ }^{\circ} \mathrm{C}$ and Con $4{ }^{\circ} \mathrm{C}$ groups (Fig. S3f-h). These data verified that cold temperature, rather than overfeeding, induced changes in gut microbiota.

\section{Cold microbiota alters gut-brain axis by activating CAMP-PKA-pCREB pathway}

To investigate the role of gut microbiota in mediating host metabolic phenotypes, we transplanted cecal microbiota from warm or cold-acclimated voles to bacterial-restricted voles (Fig. 4a). During the antibiotic treatment, body mass did not change significantly (repeated measures ANOVA, $F_{3,20}=1.416, p=0.267$, Fig. S4a), but food intake showed a significant decrease compared to that of control (repeated 
a

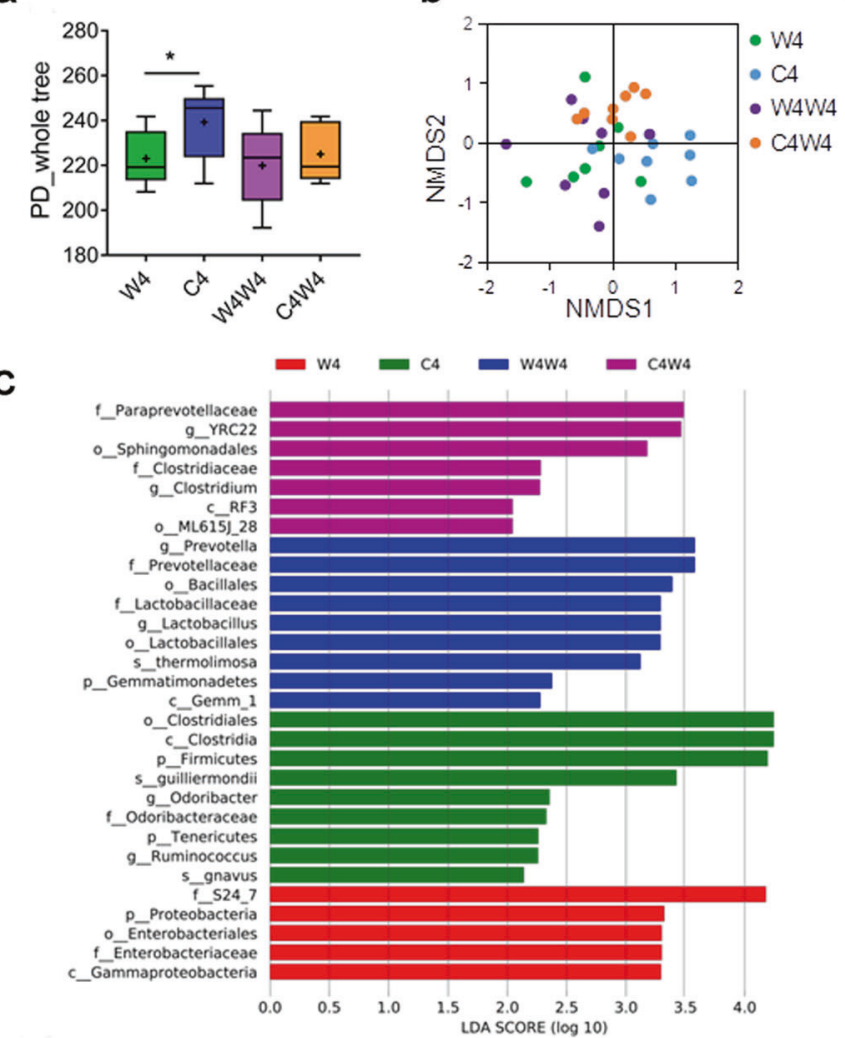

d

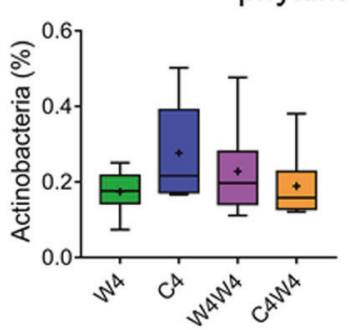

Relative abundance at the phylum level (\%)
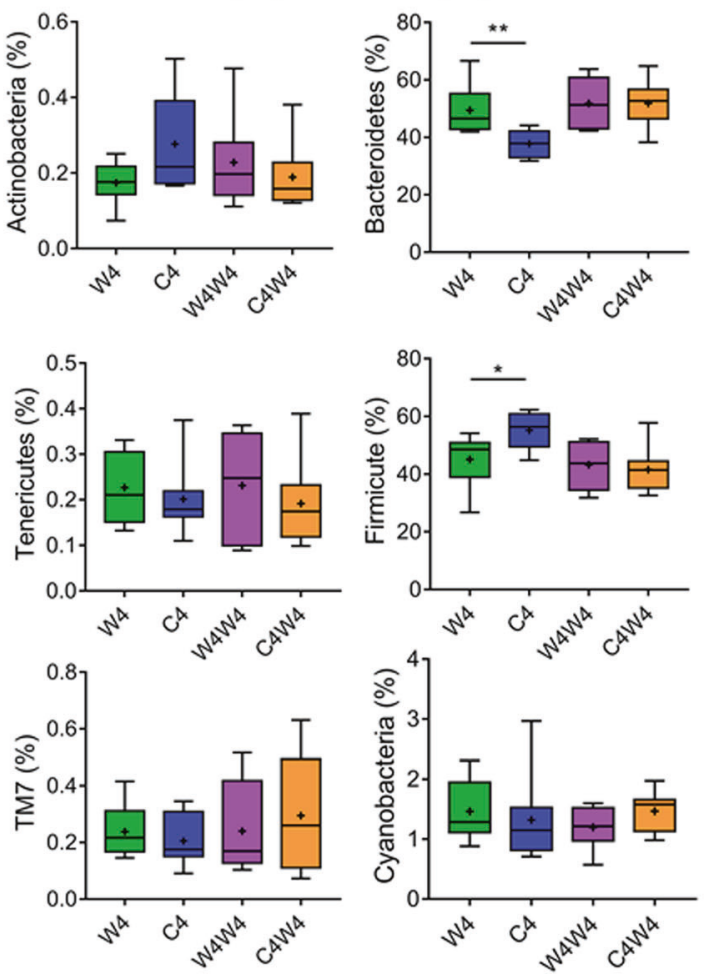

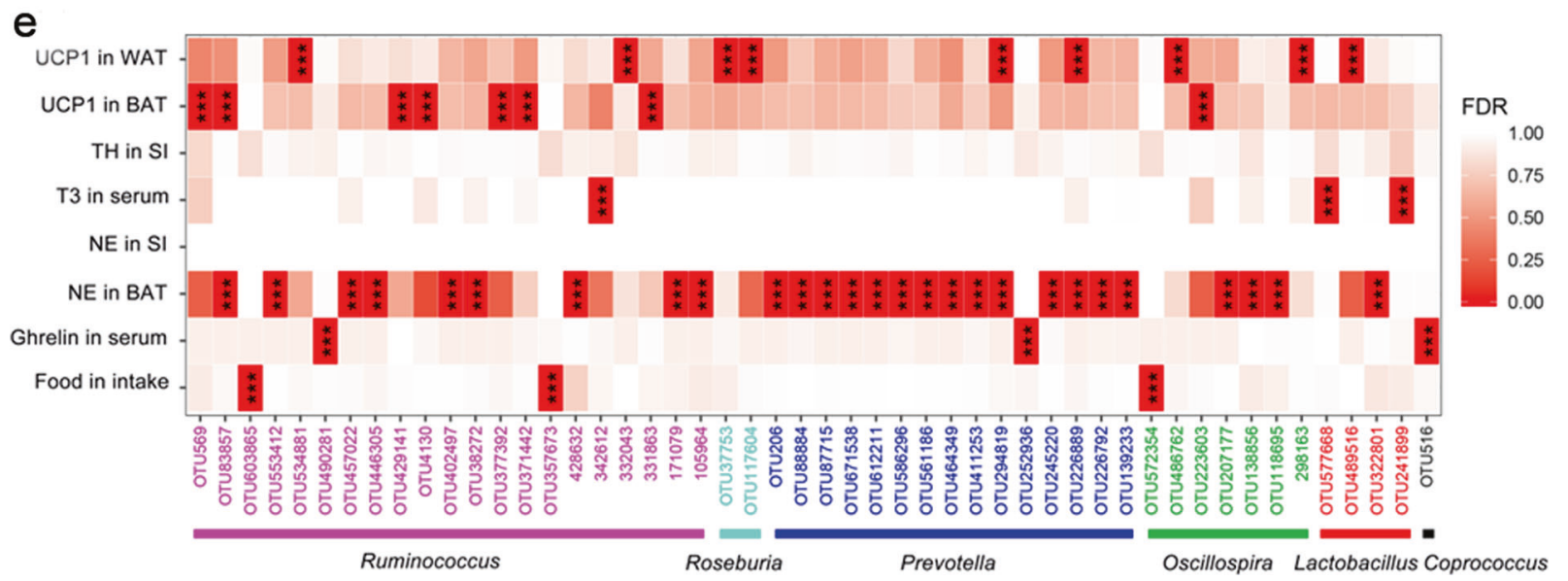

Fig. 2 Cold and rewarming shape diversity and composition of cecal microbiota. a Alpha diversity (PD_whole tree) of bacterial communities across groups ( $n=8$ per group, independent $t$-test). b NMDS plot based on unweighted UniFrac distance metrics representing the differences in fecal microbial community structure in different groups (ANOSIM). c LDA scores of the differentially abundant taxa enriched in microbiota from all the groups (taxa with LDA score $>2$ and a significance of $a<0.05$ are shown). d Abundance represented as the proportions of OTUs classified at the phylum rank. e Heatmap showing the correlation between specific OTUs and physiological measurements. The OTU IDs with only numbers were from Greengenes database, whereas those with the letters of OTU were clustered into de novo OTUs. NE norepinephrine, SI small intestine, TH tyrosine hydroxylase, UCP1 uncoupling protein 1, BAT brown adipose tissue, WAT white adipose tissue. Data are means \pm SEM $(n=7$ per group). $* p<0.05, * * p<0.01$ measures ANCOVA, $F_{3,19}=9.539, p<0.001$, Fig. S4b). After CMT, both body mass $\left(F_{3,20}=3.824, p=0.026\right.$, Fig. S4a) and food intake $\left(F_{3,19}=14.583, p<0.001\right.$, Figs. $4 \mathrm{~b}$ and $\mathrm{S} 4 \mathrm{~b}$ ) showed significant group differences, and food intake in the R-C4 group was higher than that in the RW4 group. Serum ghrelin (the des-acylated form) in the Ab group was lower than in the other groups $\left(F_{3,20}=6.221\right.$, $p=0.004$, Fig. $4 \mathrm{c})$. The RMR $\left(F_{3,19}=6.282, p=0.004\right.$, Fig. $4 d)$ and NST $\left(F_{3,19}=4.032, p=0.022\right.$, Fig. S4c) in the R-C4 group was slightly higher than in the R-W4 group. Serum ghrelin was positively correlated with food intake $(R=0.718, p<0.001$, Fig. S4d $)$ and NST $(R=0.419, p=$ 
a

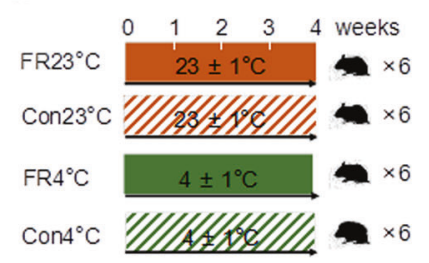

e

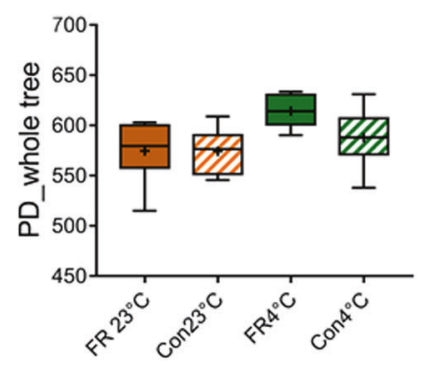

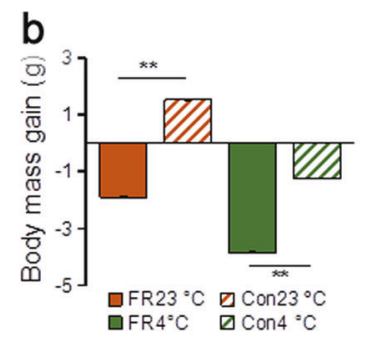

f

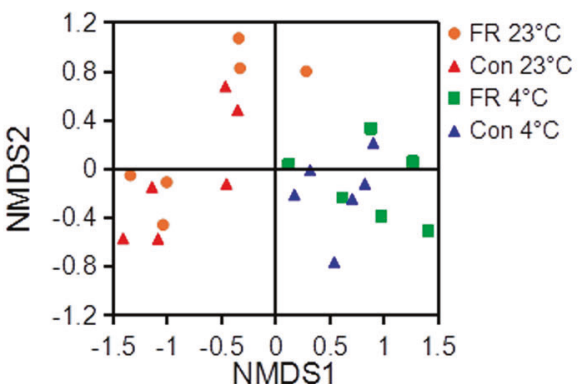

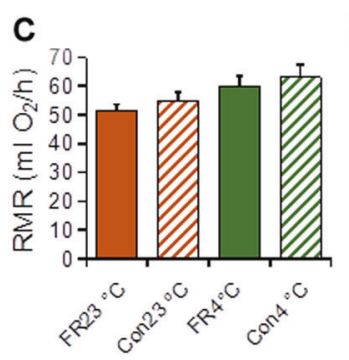

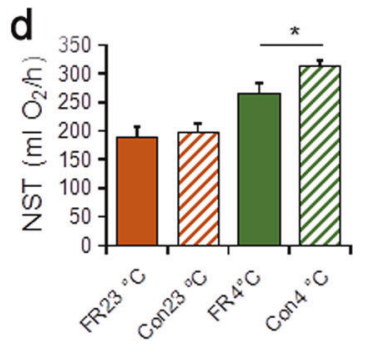

g

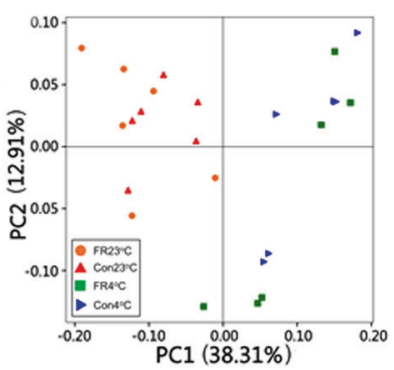

Fig. 3 Cold temperature rather than overfeeding alters bacterial diversity and composition. a The design paradigm. b Body mass gain after acclimation. c Resting metabolic rate (RMR). d Nonshivering thermogenesis (NST). e Alpha diversity measurement (Observed OTUs) of bacterial communities across groups. f, $\mathbf{g}$ NMDS and PCoA plots based on weighted UniFrac distance metrics analysis of OTUs (ANOSIM). Each symbol represents a single sample of cecal contents. Data are means \pm SEM ( $n=6$ per group, two-way ANOVA with Tukey's post hoc tests except $\beta$ diversity). $* p<0.05,{ }^{*} p<0.01$
0.042, Fig. S4e). R-C4 voles showed higher UCP1 expression in BAT compared to other groups $\left(F_{3,20}=\right.$ 11.71, $p<0.001$, Fig. 4e). NE levels of the R-C4 group were significantly higher in the BAT $\left(F_{3,20}=7.158, p=\right.$ $0.002)$ and the small intestine $\left(F_{3,20}=9.371, p<0.001\right)$, but not in the hypothalamus (Fig. $4 \mathrm{f}-\mathrm{h}$ ) compared to the other three groups. CAMP-activated PKA expression in the small intestine of the R-C4 group was higher than in the other groups $\left(F_{3,20}=7.009, p=0.002\right.$, Fig. $\left.4 \mathrm{i}\right)$. The pCREB/ CREB ratio in the small intestine of the R-C4 group was significantly higher than of the $\mathrm{Ab}$ group, but only slightly higher than the R-W4 and control groups $\left(F_{3,20}=3.751\right.$, $p=0.028$, Fig. 4j). FFAR2 expression was higher in the RC4 group than in the R-W4 group in the small intestine $\left(F_{3,20}=4.242, p=0.018\right.$, Fig. $\left.4 \mathrm{k}\right)$ and BAT $\left(F_{3,20}=8.301\right.$, $p=0.001$, Fig. 41). ELISA data showed that $\operatorname{cAMP}\left(F_{3,20}=\right.$ $0.656, p=0.589$, Fig. S4f $)$ and FFAR2 levels $\left(F_{3,20}=\right.$ $0.580, p=0.635$, Fig. S4g) in the small intestine did not show significant differences among groups. Shifts in the recipient cecal microbiota were confirmed by transplant. Antibiotic treatment $(\mathrm{Ab})$ reduced alpha diversity, indicated by PD-whole tree, observed OTUs, Chao1, Shannon Index, and Simpson Index ( $p<0.01$, Figs. $4 \mathrm{~m}$ and S4h, Table S4). For $\beta$ diversity based on unweighted (ANOSIM, $R=0.641$, $p=0.001$, Fig. $4 \mathrm{n}$ ) and weighted (ANOSIM, $R=0.316$, $p=0.001$, Fig. S4i) UniFrac distance by NMDS, there was a significant difference among groups and the R-C4 group was completely separated from the R-W4 and control groups. LEfSe methods identified the different microbial communities in the four groups (Fig. S4j, k). The Ab group showed a higher proportion of Bacteroidetes $\left(F_{3,20}=\right.$ $15.560, p<0.001)$ and lower proportions of Firmicutes $\left(F_{3,20}=11.953, p<0.001\right)$ and Actinobacteria $\left(F_{3,20}=\right.$ $11.953, p<0.001)$ than the other groups, and the proportion of Tenericutes in the R-C4 group was higher than that in the other groups $\left(F_{3,20}=4.681, p=0.012\right)$ (Fig. S41). Microbiota transplant also resulted in significant changes in the concentrations of SCFAs among groups (Fig. 4o). The concentrations of acetic acid $\left(F_{3,20}=44.587, p<0.001\right)$, butyric acid $\left(F_{3,20}=20.375, p<0.001\right)$, isobutyric acid $\left(F_{3,20}=5.862, p=0.005\right)$, and isovaleric acid $\left(F_{3,20}=\right.$ $14.391, p<0.001)$ in the R-C4 group were higher than those in the R-W4 and Ab groups (Fig. 4o).

To validate the role of gut microbiota in mediating host metabolic phenotypes through the $\beta 3$-adrenoceptor and cAMP-PKA pathways, we injected a PKA inhibitor (H89) or $\beta 3$-adrenoceptor antagonist (SR 59230A) into voles transplanted with cold-exposed microbiota (Fig. 5a). There were no differences in body mass between the four groups $(p>0.05$, Fig. S5a). NST in the R-C4-Saline group was significantly higher than in the other groups, and both H89 and SR59230A decreased NST compared to the R-W4 group $\left(F_{3,27}=148.565, p<0.01\right.$, Figs. $5 b$ and S5b). UCP1 expression in BAT was higher in the R-C4-Saline group than in the other groups $\left(F_{3,28}=8.832, p<0.001\right.$, Fig. $\left.5 \mathrm{c}\right)$. Both H89 and SR59230A induced decreases in cAMP- 


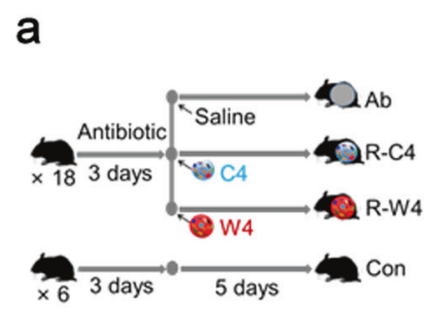

e

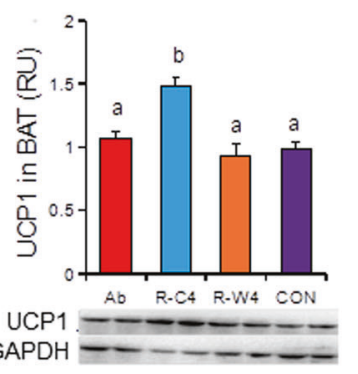

i

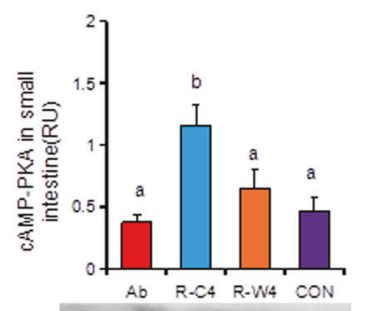

PKA GAPDH

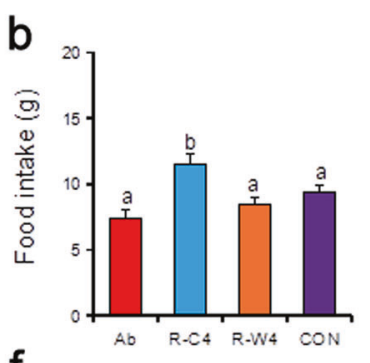

f
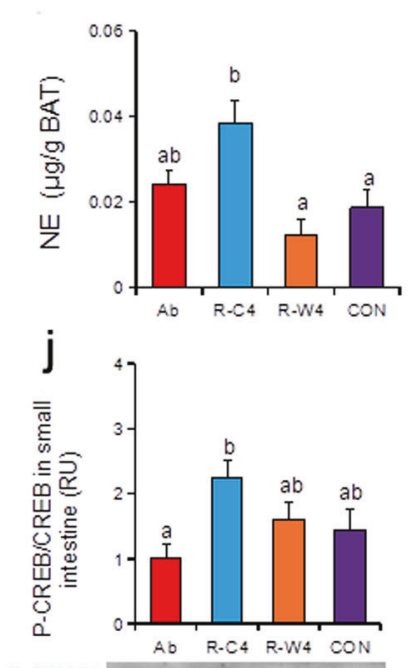

P-CREB $\ldots \ldots \ldots \ldots$

CREB

GAPDH $\mathrm{m}$

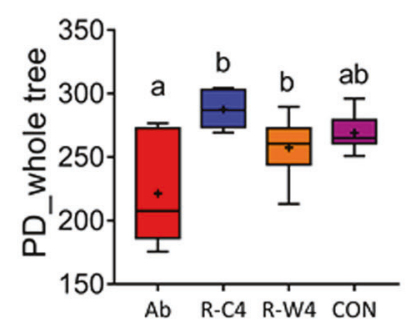

n

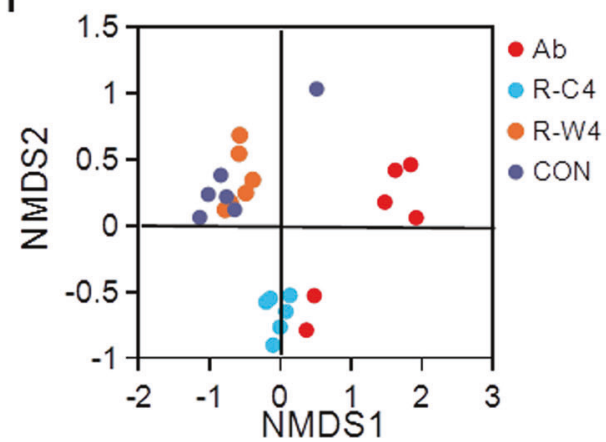

Fig. 4 Cecal microbiota transplant alters metabolic phenotypes, neurotransmitters, and bacterial diversity and composition. a The design paradigm. b Food intake, $\mathbf{c}$ serum ghrelin, $\mathbf{d}$ resting metabolic rate (RMR), and e uncoupling protein 1 (UCP1) expression in brown adipose tissue (BAT) in antibiotic $(\mathrm{Ab})$, recipients with cold microbiota (R-C4), recipients with warm microbiota (R-W4), and control groups. f Norepinephrine (NE) concentration in BAT, $\mathbf{g}$ small intestine, and $\mathbf{h}$ hypothalamus (hypo). i The cAMP-activated PKA expression in the small intestine. j Phospho-

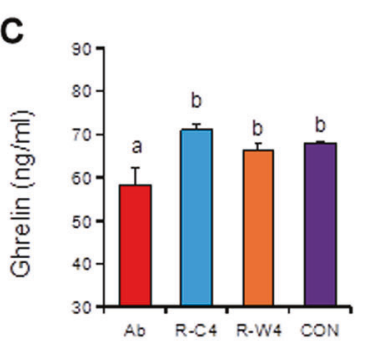

g

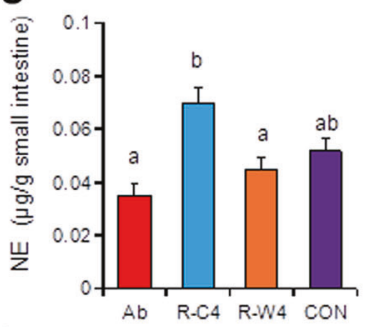

k

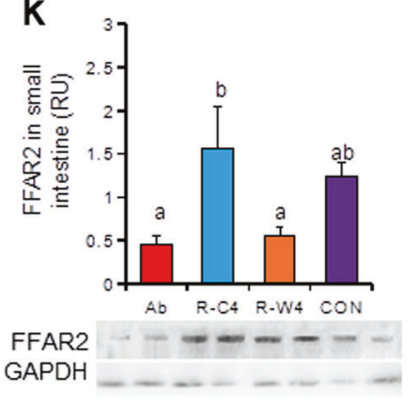

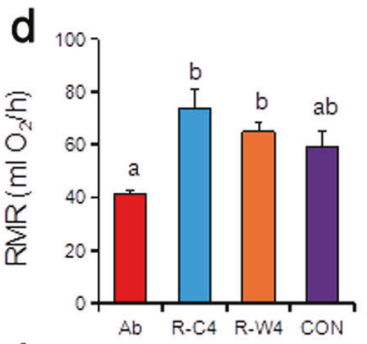

h
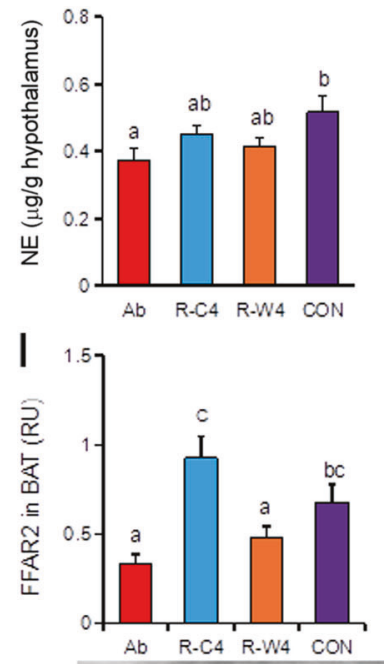

FFAR2 $=a m=-m=m=m$

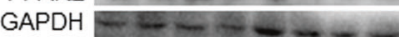
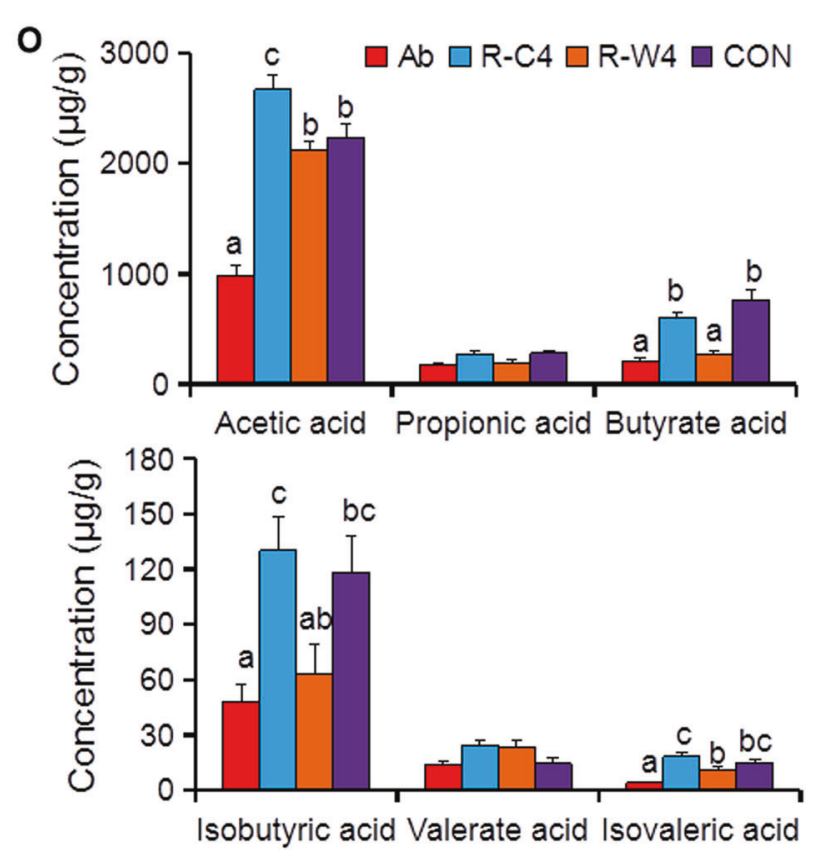

CREB/CREB in the small intestine. $\mathbf{k}$ The FFAR2 expression in the small intestine and $\mathbf{l}$ BAT. $\mathbf{m}$ The comparison of alpha diversity measurement (PD_whole tree) of bacterial communities across groups. n NMDS plot based on unweighted UniFrac distance metrics shows the microbial community structure of samples from different groups (ANOSIM). o The concentrations of six short-chain fatty acids (SCFAs) in cecal contents. Data are means \pm SEM $(n=6$ per group, one-way ANOVA with Tukey's post hoc tests except $\beta$ diversity). $* p<0.05, * * p<0.01$ 
a

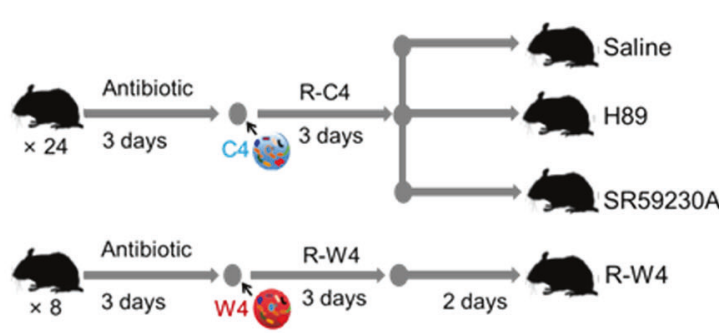

d

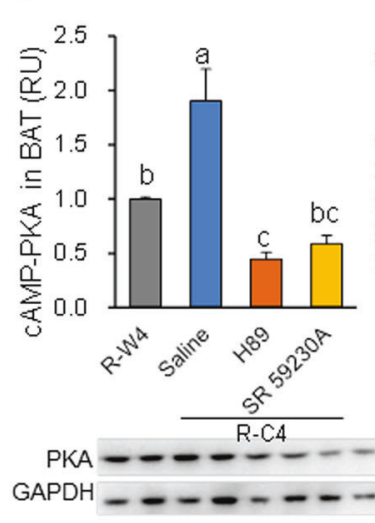

e

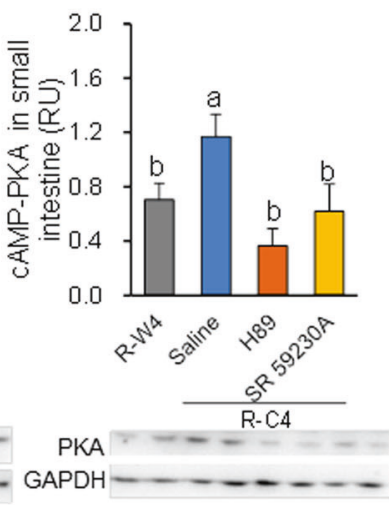

Fig. 5 Cold microbiota promotes thermogenesis via the cAMP-PKA-pCREB pathway. a The design paradigm. b Nonshivering thermogenesis (NST). c Uncoupling protein 1 (UCP1) expression in brown adipose tissue (BAT). d cAMP-activated PKA expression in BAT and e small intestine. f Phospho-CREB/CREB in b

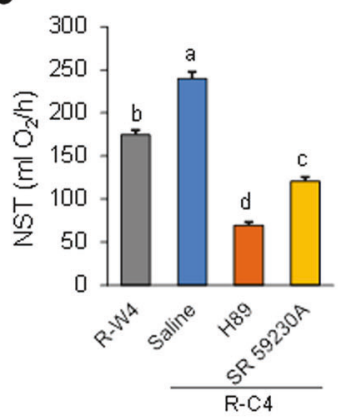

f

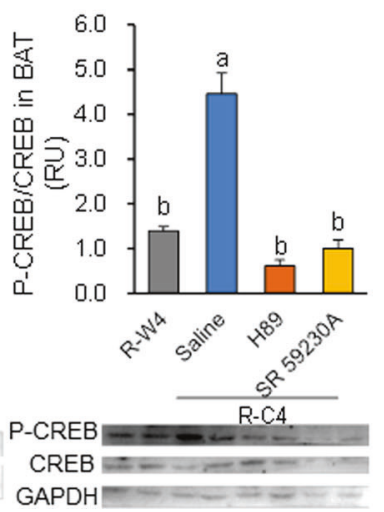

C

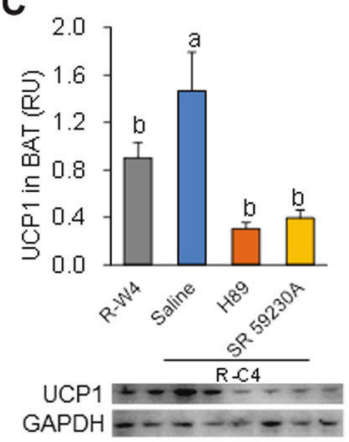

g

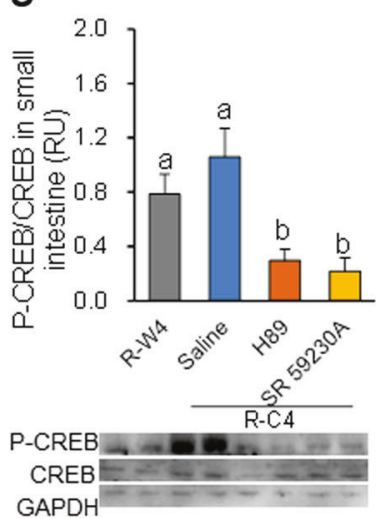

BAT and $\mathbf{g}$ small intestine. Data are means $\pm \operatorname{SEM}(n=8$ per group, except phospho-CREB/CREB ( $n=7$ per group), one-way ANOVA with Tukey's post hoc tests). Different superscript letters indicate significant differences among different groups $(p<0.05)$

group than that in the control group $\left(F_{1,14}=14.892, p=\right.$ 0.002 , Fig. 6d), but, after 3-week recovery, there were no differences between the control and NE-R21 groups. UCP1 expression in BAT of the NE group was higher than the control group $\left(F_{3,24}=4.696, p=0.01\right.$, Fig. 6e). NE manipulation resulted in increases in cAMP-activated PKA $\left(F_{3,20}=3.092, p=0.05\right.$, Fig. 6f $)$ and p-CREB expressions $\left(F_{3,24}=5.122, p=0.007\right.$, Fig. $\left.6 \mathrm{~g}\right)$ in the small intestine compared to controls, even after a recovery period. cAMP levels both in the small intestine $\left(F_{3,26}=5.013, p=0.007\right.$, Fig. S6a) and BAT $\left(F_{3,17}=3.918, p=0.027\right.$, Fig. S6b) increased significantly in the NE-R7 group compared to those in the other groups. Analysis of 16S rRNA gene sequencing showed that there were no differences in alpha diversity among the four groups (Fig. 6h, S6c, Table S5), but NE manipulations followed by a recovery period for 7 days (NE-R7) significantly influenced cecal microbiota structure ( $\beta$ diversity based on unweighted UniFrac distance, ANOSIM, $r=0.197, P=0.001$, Fig. 6i, but with no difference based on weighted UniFrac distance, $r=0.052, P=0.090$, Fig. S6d). LEfSe methods identified the discriminative features in the microbiota of the four groups (Figs. $6 \mathrm{j}$ and S6e). 
a

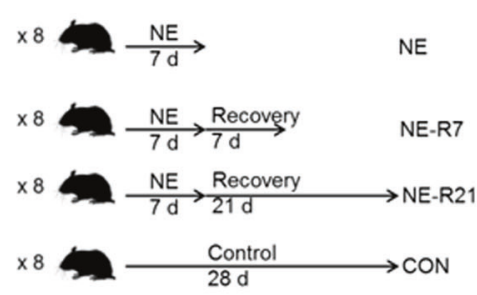

d

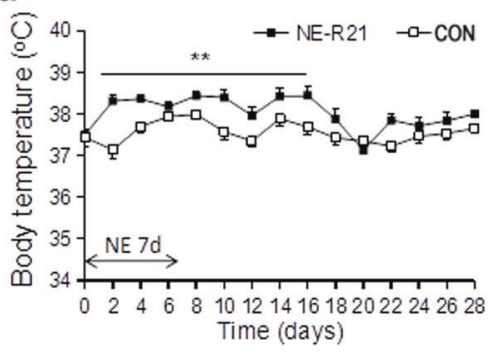

h

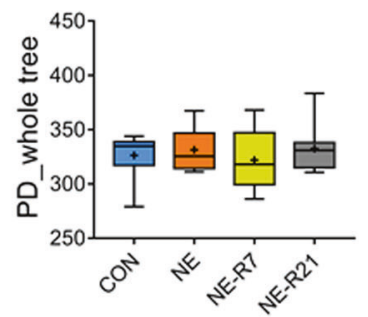

k

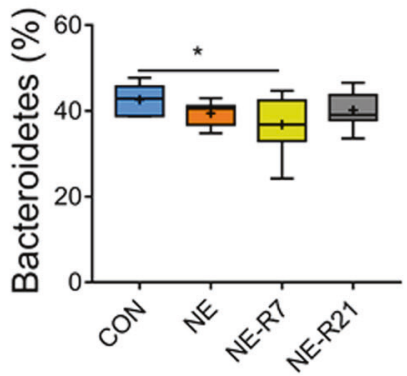

Fig. 6 Norepinephrine (NE) manipulation affects energy metabolism and gut microbiota. a The design paradigm. b Body mass. c Food intake. d Body temperature. e Uncoupling protein 1 (UCP1) expression in brown adipose tissue (BAT). f The cAMP-activated PKA expression in the small intestine. $g$ Phospho-CREB/CREB in the small intestine. $\mathbf{h}$ The comparison of alpha diversity measurement (PD_whole tree) of bacterial communities among groups. i NMDS plot based on unweighted UniFrac distance representing the differences in the microbial community structures of samples from different groups. j Differential bacterial taxonomy selected by LEfSe analysis with LDA

At the phylum level, the proportion of Elusimicrobia increased in the NE-R7 group and Tenericutes increased both in NE and NE-R7 groups compared to the control and NE-R21 groups $(p<0.01$, Figs. $6 \mathrm{k}$ and S6f). Compered to controls, NE increased the abundance of Facklamia $(p=$

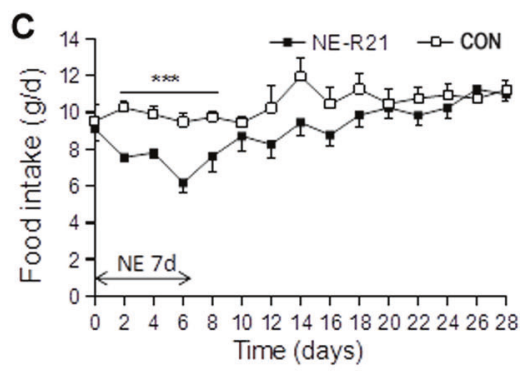

f
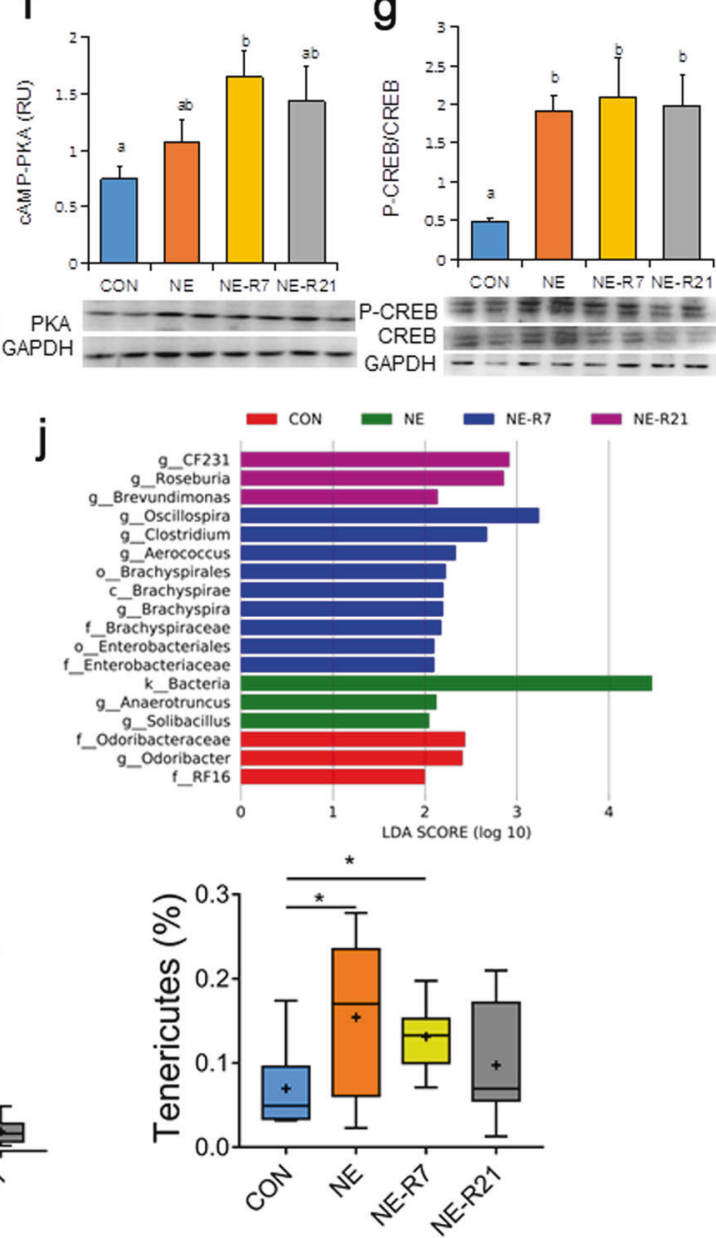

score $>2$ in microbiota. $\mathbf{k}$ Abundance represented as the proportions of OTUs classified at the phylum level. Data are means \pm SEM $(n=8$ per group). Body mass, food intake, and body temperature were analyzed by repeated measures ANOVA, $\beta$ diversity by ANOSIM, and others by one-way ANOVA with Tukey's post hoc tests. NE, NE manipulation for 7 days; NE-R7, NE manipulation for 7 days and then recover for 7 days; NE-R21, NE manipulation for 21 days and then recover for 21 days. Different superscript letters indicate significant differences among different groups $(p<0.05)$. $* P<0.05$

$0.035)$ and Erwinia $(p=0.035)$; the NE-R7 group increased Propionibacterium $(p=0.035)$, Devosia $(p=0.035)$, Erwinia $(p=0.027)$, and Clostridium $(p<0.01$, Table S6). This study illustrates that NE had long-lasting impacts on gut microbiota and metabolic phenotypes. 
Fig. 7 The paradigm summarizing the crosstalk between gut microbiota and norepinephrine (NE) via the cAMP signaling pathway in mediating host energetics and thermogenesis during cold acclimation. BAT brown adipose tissue, cAMP cyclic adenosine monophosphate, FFAR2 free fatty acid receptor 2, p-CREB phosphorylation of cAMP-response elementbinding protein, PKA protein kinase A, SCFAs short-chain fatty acids, UCP1 uncoupling protein 1, WAT white adipose tissue

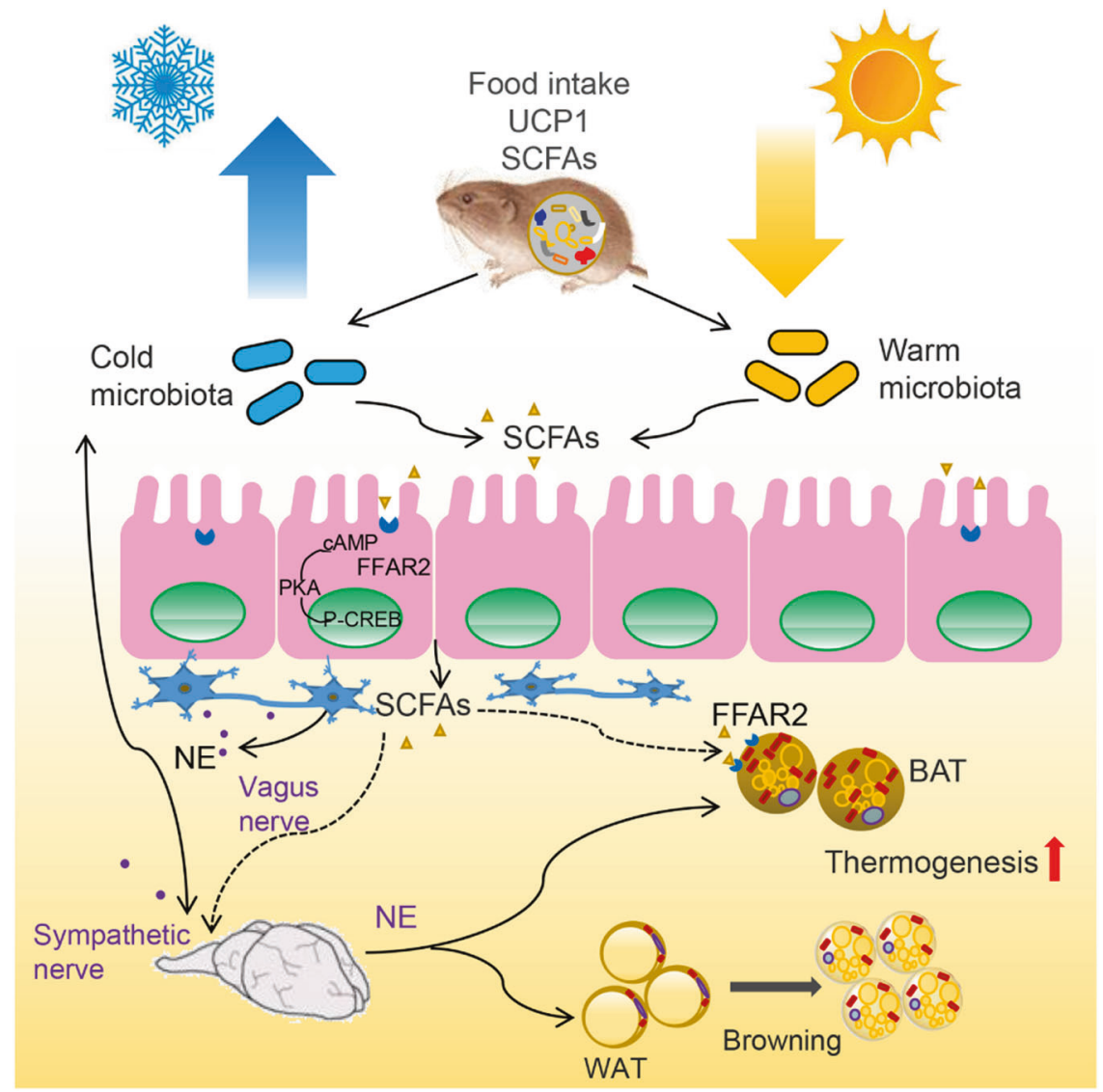

\section{Discussion}

The gut microbial community is shaped by physiological, dietary, and social influences, that ultimately affect host metabolism [15, 29, 32]. In the present study, we investigated the bidirectional pathway between gut microbiota and $\mathrm{NE}$ in regulating energy intake and thermogenesis. We found that the composition and structure of cecal microbiota were directly influenced by cold temperature exposure, and there were correlations between cecal microbiota, monoamine neurotransmitter levels, and thermogenesis. Further, cecal microbiota transplantation confirmed that coldexposed microbiota increased the concentrations of SCFAs and their receptor (FFAR2), activated cAMP-PKA-pCREB signaling, increased NE concentrations in the small intestine and BAT, and increased serum ghrelin levels, thus leading to increased energy intake and thermogenesis of the host. Both a $\beta 3$-adrenoceptor antagonist and a PKA inhibitor could block cold microbiota-induced increases in NST and UCP1 expression in BAT. In addition, NE modulated microbial composition via upregulation of the cAMP-PKA-pCREB pathway in the gut. These data suggest that gut microbiota-NE interactions through the cAMP-PKApCREB pathway mediated host energetics and thermogenesis during cold acclimation (Fig. 7).
There are many physiological and behavioral strategies for small mammals to survive cold environments in the field. Both males and females increase food intake, RMR, NST, and UCP1 expression in BAT during cold acclimation $[2,3]$. The present study showed that serum des-acylated ghrelin levels increased during cold acclimation. Two major endogenous forms (acylated and des-acyl) of ghrelin exist in the stomach, small intestine, and hypothalamus [33]. The acylated form of ghrelin is essential for stimulating feeding and growth hormone (GH) release by acting on the $\mathrm{GH}$ secretagogue type 1a receptor (GHS-R1a), and the desacylated form circulated in far greater amounts than the acylated form, stimulated cell proliferation, adipogenesis, and counteracted the metabolic action of acylated form of ghrelin [34]. The des-Acyl ghrelin could also induce food intake independent of the GHS-R1a [35]. Together with other previous data [10], the increase in serum des-Acyl ghrelin levels contributes to an increased appetite and thermogenesis under cold conditions. Cold-induced increases in RMR and NST are mainly activated by increased secretion of thyroid hormones and NE [4]. In our study, the browning in white adipocytes and increased UCP1 expression both in BAT and WAT were associated with increased serum $\mathrm{T} 3$ levels, increased T3/T4 ratios, and increased $\mathrm{TH}$ and NE expression in BAT and WAT. The 
increased NE levels, along with increased 5-HT and dopamine turnover rates in the small intestine during cold acclimation could stimulate ghrelin secretion [36], leading to enhanced energy intake and thermogenesis. These monoamines in the gut are produced by certain strains of bacteria and the ENS [25, 37], as well as from sympathetic fibers in the gut wall. All these data suggest that monoamine neurotransmitters, such as NE, are involved in regulating energy intake and thermogenesis during cold acclimation.

Correlative evidence has revealed a metabolic interaction between gut microbial communities and the host, and gut bacteria play an important role in the regulation of brown and beige adipose tissues [38, 39]. The present study revealed that the most abundant phyla in the cecum of Brandt's voles are Firmicutes and Bacteroidetes, consistent with other mammals, such as rabbits [40], mice [38], pikas [41], and humans [42]. Firmicutes and Bacteroidetes are mainly responsible for food fermentation in the cecum [43]. At the family level, Ruminococcaceae and S24-7 were the dominant identified families in the voles. Different from mice, Prevotella and Ruminococcus were the dominant identified genera in the voles because the genera Prevotella, Oscillospira, Ruminococcus, and Treponema were associated with the fermentation of plant-based food and considered fiber-degrading bacteria [44-46]. Ruminococcus exists in the rumen of most ruminants and in the cecum of most herbivores, such as the $R$. bromiihe which can degrade starch and R. flavefaciens which can degrade cellulose [47].

Cold acclimation led to significant changes in microbiota composition, especially increased Firmicutes to Bacteroidetes ratios. Many studies show that temperature affects an animal's gut microbiota. Plateau pikas (Ochotona curzoniae) have higher microbial diversity and cellulase activity than low altitude pikas (Ochotona daurica), showing that cold and hypoxia environments affect gut microbiota [41]. Cold-exposed mice have altered microbiota composition, including a higher abundance of Proteobacteria, a reduction in Bacteroidetes and Akkermansia muciniphila, an increase in Firmicutes [48], and the Verrucomicrobia phylum is almost absent [38]. Our results showed that the voles increased the proportions of Odoribacter, Paraprevotella, Clostridium, [Ruminococcus], and Helicobacter after cold acclimation. Brandt's voles are strict herbivores, which may cause these different changes in gut microbial composition in response to cold temperatures. Diet is an important factor for affecting gut microbiota [49], and the change in gut microbiota during cold acclimation can be induced by increased food intake instead of cold temperatures. Using pair-fed manipulations, we confirmed that cold temperatures still altered the diversity and composition of cecal microbiota even after controlling for food intake. Our previous study showed that cold-acclimated voles have $1{ }^{\circ} \mathrm{C}$ lower core body temperature compared to voles in warm rooms [29]. Cold exposure induces a decrease in gut temperature and an increase in NE release, and thus leading to an increased conversion of cholesterol to bile acids, which may influence the gut microbiome $[29,50]$. These findings indicate that cold temperature, rather than overfeeding, results in the alteration of gut micriobiota during cold acclimation.

Using correlation analyses, we found that at the genus level, Oscillospira and Ruminococcus were correlated with food intake, and Lactobacillus, Oscillospira, Prevotella, Roseburia, and Ruminococcus were correlated with BAT thermogenesis. These correlations between cecal microbiota and host thermogenesis were further verified by CMT in the present study. Cold-exposed microbiota transplant resulted in increased food intake, NE, and UCP1 expression in BAT in Brandt's voles. In mice fed high-fat food and during cold acclimation, Firmicutes changes are positively linked with ucp 1 expression and negatively linked with obesity [51]. Li et al. [52] found that an every-other-day fasting treatment can activate beige fat, and this is most likely achieved through re-shaping of gut microbiota [52]. Germ-free mice transplanted with gut microbiota from mice living in cold environments show white fat browning [38], reduced fat mass and adiposity, and significantly higher expression of ucpl and Dio2 mRNA and protein in BAT [48]. SCFAs, the principal end-products of microbial fermentation of dietary fiber can supply $5-10 \%$ of energy requirements for humans [53]. SCFAs can also be as signal molecules by acting on G-protein-coupled receptors GPR41 (renamed free fatty acid receptor 3, FFAR3) and GPR43 (FFAR2) during appetite regulation, thermoregulation, and energy homeostasis [54]. These receptors are coexpressed with glucagon-like peptide 1 (GLP-1), peptide YY (PYY), or ghrelin in enteroendocrine cells and are also expressed in other various tissues and cell types such as the lung, spleen, adipose tissue, testis, and peripheral blood mononuclear cells [55-58]. Increasing evidence indicate that acetate, butyrate, and propionate can regulate appetite and activate BAT by increasing GLP-1/PYY or ghrelin secretion $[21,59,60]$. The signaling mechanisms underlying the regulatory roles of SCFAs have been mostly studied in vitro, which show that butyrate can regulate colonocyte proliferation and apoptosis as well as the progesterone and estradiol biosynthesis via cAMP pathways in cultivated cell lines $[60,61]$. Pro-inflammatory cytokines, such as IFN- $\gamma$, in macrophages can also activate the cAMP-PKA-pCREB signaling pathway [62]. We found that in comparison to warm temperature-exposed microbiota, cold temperatureexposed microbiota increased SCFA (particularly butyric acid) concentrations, FFAR2 expression, NE levels in the small intestine and BAT, and activated the cAMP-PKA-pCREB signaling pathway in the small intestine in vivo. We also showed that cold microbiota 
transplant-induced BAT thermogenesis was significantly attenuated by both a $\beta 3$-adrenoceptor antagonist and a PKA inhibitor. The cAMP-PKA-pCREB signaling pathway may be co-activated through increased SCFAs, inflammatory cytokines, UCP1, and lipolysis during cold acclimation [60-64]. All these data indicate that cold-induced thermogenesis is regulated by gut microbiota and the gut-brain axis via co-activated cAMP signaling pathways. FFAR2 expression in BAT was also increased in voles transplanted with cold temperature-exposed microbiota compared to warm temperature-exposed transplant voles, implying that circulating SCFAs acting on FFAR2 in BAT may also directly regulate BAT thermogenesis. The specific mechanism underlying how SCFAs regulate thermogenesis need to be further investigated. The voles are herbivore and need to rely on cecal bacteria to digest the high-fiber food. For animal safety, we chose the antibiotic treatment regimen for short time. We should point out that 3-day treatment regimen may impact the transplant effect in comparison to longer antibiotic treatments which induce a more substantial bacterial knockdown. In addition, we acknowledge the technical limitations for protein quantification in this present study. Multiple protein/transcript quantitative methods to be used would validate these results.

Increasing evidence suggests that the communication between gut microbiota and the host brain is bidirectional. Monoamine neurotransmitters are most likely involved in the communication between the microbiota and the brain by sensing SCFAs and/or gut hormones [25, 65, 66]. Microbial endocrinology studies have shown that a number of bacteria colonized in the gastrointestinal tract, such as Lactobacillus, Bifidobacterium, and Escherichia strains, can produce neurotransmitters including 5-HT, dopamine, NE, and gamma-aminobutyric acid (GABA), which can then bind to host receptors and are essential in regulating ENS development and neurophysiological and behavioral changes of the host [25, 66, 67]. In the opposite direction, host neurotransmitters, such as NE, can alter proliferative activity of microbiota and increase virulent properties of some strains of enteric pathogens in vitro [68]. Substantially less work has been done to determine whether host-derived neurotransmitters can influence gut microbiota. Our present data show that NE manipulation induced long-lasting alterations in the composition and structure of fecal microbiota, and the cAMP signaling pathway in the small intestine was activated in the NE-R7 group, suggesting that host neurotransmitters can also affect gut bacterial diversity. In addition, NE manipulations resulted in long-term, physiological changes, including increased body temperature and UCP1 expression in BAT, and decreased food intake and body mass. Taken together, these finding suggest that crosstalk between gut microbiota and NE mediated host metabolism and thermogenesis.

\section{Conclusion}

Air temperature varies with annual cycles, and small mammals adjust their metabolic physiology and microbial symbionts accordingly. The present study, which integrates microecology and physiological ecology, illustrates the relationship and regulatory pathways between host metabolic phenotypes and gut microbiota during cold acclimation in male Brandt's voles. Our data clearly show that cold acclimation initiated a wide range of physiological changes, such as increased food intake, increased levels of UCP1, ghrelin, and $\mathrm{TH}$, and an increase in turnover rates of DA and 5-HT in different body tissues. Cold acclimation also caused a significant alteration in cecal microbiota, which was accompanied by an increase in total concentration of SCFAs in cold-acclimated voles. We also confirmed that cold temperature, rather than overfeeding, influenced microbiota structure. Further, CMT into antibiotic-treated voles appeared to recapitulate the observed cold phenotype, thus providing a link between physiological changes in response to the cold and modulation of this adaptation by gut microbiota. Moreover, the increases in NE appeared to be driven by transplanted microbiota, and NE increases lead to a higher expression of UCP1, cAMP-PKA, and pCREB, as well as changes in cecal microbiota. These findings indicate that microbiota-gut-brain interactions via cAMP signaling pathways mediate host energetics and thermogenesis during cold acclimation. This sheds a considerable new light on the mechanisms of communication between the host and microbial symbionts used to improve the survival of small mammals in seasonal environments. Male and female voles have a similar metabolic adaptation to cold [2, 69], photoperiod [2, 27], and food quality [70, 71]; however, sex differences in the gut microbiota and its impact on cold tolerance have not been investigated. Further comparative studies in mammalian microbiota responses to diverse environmental and social factors between sexes are needed to fully illustrate the crucial cues that drive the coevolution of host and microbial symbionts.

\section{Data availability}

Raw sequence data are deposited in the NCBI Sequence Read Archive under accession PRJNA555499, PRJNA555506, PRJNA555511, and PRJNA555551.

Acknowledgements We appreciate the very helpful and constructive comments and suggestions from the three anonymous reviewers and the editor for improving the manuscript. We are grateful to Prof. Zuoxin Wang and Meghan Donovan from Department of Psychology and Program in Neuroscience, Florida State University for the comments and careful editing. We thank Jianfeng Wang from Beijing Nebula Medical Laboratory Co., Ltd. for helps in 16S rRNA gene sequencing and analyses. We also thank Prof. Jianxu Zhang and Dr. 
Yaohua Zhang for supplying some chemicals and technique, and thank all the members of Animal Physiological Ecology Group for their help in the experiments and discussions. This research was supported by the National Natural Science Foundation of China (Nos. 31770440 and 31772461), and the Beijing Natural Science Foundation (5172024).

Author contributions $\mathrm{X}-\mathrm{YZ}$ and D-HW designed the studies. T-BB and $\mathrm{X}-\mathrm{YZ}$ conducted the experiments. T-BB, X-YZ, JW, and X-WQ performed the measurements of SCFAs and neurotransmitters. T-BB and $\mathrm{X}-\mathrm{YZ}$ analyzed the data. TBB, $\mathrm{X}-\mathrm{YZ}$, and $\mathrm{KD}$ made the figures. T-BB, X-YZ, and D-HW wrote the paper. All authors read and approved the final manuscript.

\section{Compliance with ethical standards}

Conflict of interest The authors declare that they have no conflict of interest.

Publisher's note: Springer Nature remains neutral with regard to jurisdictional claims in published maps and institutional affiliations.

\section{References}

1. McNab BK. The physiological ecology of vertebrates. Ithaca: Cornell University Press; 2002.

2. Li XS, Wang DH. Photoperiod and temperature can regulate body mass, serum leptin concentration, and uncoupling protein 1 in Brandt's voles (Lasiopodomys brandtii) and Mongolian gerbils (Meriones unguiculatus). Physiol Biochem Zool. 2007;80: 326-34.

3. Zhang XY, Wang DH. Energy metabolism, thermogenesis and body mass regulation in Brandt's voles (Lasiopodomys brandtii) during cold acclimation and rewarming. Horm Behav. 2006; 50:61-9.

4. Cannon B, Nedergaard J. Brown adipose tissue: function and physiological significance. Physiol Rev. 2004;84:277-359.

5. Heldmaier G, Steinlechner S, Rafael J. Nonshivering thermogenesis and cold resistance during seasonal acclimatization in the Djungarian hamster. J Comp Physiol B. 1982;149:1-9.

6. Nicholls D, Locke R. Thermogenic mechanisms in brown fat. Physiol Rev. 1984;64:1-64.

7. Walter GC, Phillips RJ, McAdams JL, Powley TL. Individual sympathetic postganglionic neurons coinnervate myenteric ganglia and smooth muscle layers in the gastrointestinal tract of the rat. J Comp Neurol. 2016;524:2577-603.

8. Himms-Hagen J. Thyroid hormones and thermogenesis. Mammalian thermogenesis. Netherlands: Springer; 1983.

9. Mullur R, Liu YY, Brent GA. Thyroid hormone regulation of metabolism. Physiol Rev. 2014;94:355-82.

10. Uchida Y, Nagashima K, Yuri K. Fasting or systemic des-acyl ghrelin administration to rats facilitates thermoregulatory behavior in a cold environment. Brain Res. 2018;1696:10-21.

11. Cannon B, Nedergaard J. Thyroid hormones: igniting brown fat via the brain. Nat Med. 2010;16:965-7.

12. Contreras C, Nogueiras R, Diéguez C, Rahmouni K, López M. Traveling from the hypothalamus to the adipose tissue: the thermogenic pathway. Redox Biol. 2017;12:854-63.

13. Faith JJ, McNulty NP, Rey FE, Gordon JI. Predicting a human gut microbiota's response to diet in gnotobiotic mice. Science. 2011;333:101-4.

14. David LA, Maurice CF, Carmody RN, Gootenberg DB, Button JE, Wolfe BE, et al. Diet rapidly and reproducibly alters the human gut microbiome. Nature. 2014;505:559-63.
15. Koren O, Goodrich JK, Cullender TC, Spor A, Laitinen K, Bäckhed HK, et al. Host remodeling of the gut microbiome and metabolic changes during pregnancy. Cell. 2012;150:470-80.

16. Turnbaugh PJ, Backhed F, Fulton L, Gordon JI. Diet-induced obesity is linked to marked but reversible alterations in the mouse distal gut microbiome. Cell Host Microbe. 2008;3:213-23.

17. Martinez-Guryn K, Hubert N, Frazier K, Urlass S, Musch MW, Ojeda $\mathrm{P}$, et al. Small intestine microbiota regulate host digestive and absorptive adaptive responses to dietary lipids. Cell Host Microbe. 2018;23:458-69.

18. Bäckhed F, Ley RE, Sonnenburg JL, Peterson DA, Gordon JI. Host-bacterial mutualism in the human intestine. Science. 2005;307:1915-20.

19. Crawford PA, Crowley JR, Sambandam N, Muegge BD, Costello EK, Hamady M, et al. Regulation of myocardial ketone body metabolism by the gut microbiota during nutrient deprivation. Proc Natl Acad Sci USA. 2009;106:11276-81.

20. Li Z, Yi CX, Katiraei S, Kooijman S, Zhou E, Chung CK, et al Butyrate reduces appetite and activates brown adipose tissue via the gut-brain neural circuit. Gut. 2018;67:1269-79.

21. Perry RJ, Peng L, Barry NA, Cline GW, Zhang DY, Cardone RL, et al. Acetate mediates a microbiome-brain-beta-cell axis to promote metabolic syndrome. Nature. 2016;534:213-7.

22. Donohoe DR, Garge N, Zhang X, Sun W, O'Connell TM, Bunger $\mathrm{MK}$, et al. The microbiome and butyrate regulate energy metabolism and autophagy in the mammalian colon. Cell Metab. 2011;13:517-26.

23. Psichas A, Sleeth ML, Murphy KG, Brooks L, Bewick GA, Hanyaloglu AC, et al. The short chain fatty acid propionate stimulates GLP-1 and PYY secretion via free fatty acid receptor 2 in rodents. Int J Obes. 2015;39:424-9.

24. De Vedder F, Grasset E, Holm LM, Karsenty G, Macpherson AJ, Olofsson LE, et al. Gut microbiota regulates maturation of the adult enteric nervous system via enteric serotonin networks. Proc Natl Acad Sci USA. 2018;115:6458-63.

25. Lyte M. Microbial endocrinology: host-microbiota neuroendocrine interactions influencing brain and behavior. Gut Microbes. 2014;5:381-9.

26. Yano JM, Yu K, Donaldson GP, Shastri GG, Ann P, Nagler CR, et al. Indigenous bacteria from the gut microbiota regulate host serotonin biosynthesis. Cell. 2015;161:264-76.

27. Zhao ZJ, Wang DH. Short photoperiod enhances thermogenic capacity in Brandt's voles. Physiol Behav. 2005;85:143-9.

28. Bo TB, Zhang XY, Wang DH. Effects of cold acclimation on the structure of small intestinal mucosa and mucosal immunityassociated cells in Lasiopodomys brandtii. Acta Theriol Sin. 2018;2:158-65.

29. Zhang XY, Sukhchuluun G, Bo TB, Chi QS, Yang JJ, Chen B, et al. Huddling remodels gut microbiota to reduce energy requirements in a small mammal species during cold exposure. Microbiome. 2018;6:103.

30. Wang JM, Wang DH. Comparison of nonshivering thermogenesis induced by dosages of norepinephrine from 3 allometric equations in Brandt's voles (Lasiopodomys brandtii). Acta Theriol Sin. 2006;26:84-8.

31. Dunphy-Doherty F, O'Mahony SM, Peterson VL, O'Sullivan O, Crispie F, Cotter PD, et al. Post-weaning social isolation of rats leads to long-term disruption of the gut microbiota-immune-brain axis. Brain Behav Immun. 2018;68:261-73.

32. Amato KR, Yeoman CJ, Kent A, Righini N, Carbonero F, Estrada A, et al. Habitat degradation impacts black howler monkey (Alouatta pigra) gastrointestinal microbiomes. ISME J. 2003;7:1344-53.

33. Hosoda H, Kojima M, Matsuo H, Kangawa K. Ghrelin and des-acyl ghrelin: two major forms of rat ghrelin peptide in gastrointestinal tissue. Biochem Biophys Res Commun. 2000;279:909-13. 
34. Broglio F, Gottero C, Prodam F, Gauna C, Muccioli G, Papotti M, et al. Non-acylated ghrelin counteracts the metabolic but not the neuroendocrine response to acylated ghrelin in humans. J Clin Endocrinol Metab. 2004;89:3062-5.

35. Toshinai K, Yamaguchi H, Sun Y, Smith RG, Yamanaka A, Sakurai $\mathrm{T}$, et al. Des-Acyl ghrelin induces food intake by a mechanism independent of the growth hormone secretagogue receptor. Endocrinology. 2006;147:2306-14.

36. Mundinger TO, Cummings DE, Taborsky GJ. Direct stimulation of ghrelin secretion by sympathetic nerves. Endocrinology. 2006;147:2893-901.

37. Furness JB. Types of neurons in the enteric nervous system. J Auton Nerv Syst. 2000;81:87-96.

38. Chevalier C, Stojanović O, Colin DJ, Suarez-Zamorano N, Tarallo V, Veyrat-Durebex C, et al. Gut microbiota orchestrates energy homeostasis during cold. Cell. 2015;163:1360-74.

39. Nicholson JK, Holmes E, Kinross J, Burcelin R, Gibson G, Jia W, et al. Host-gut microbiota metabolic interactions. Science. 2012;336:1262-7.

40. Velasco-Galilea M, Piles M, Viñas M, Rafel O, GonzálezRodríguez O, Guivernau M. et al. Rabbit microbiota changes throughout the intestinal tract. Front Microbiol. 2018;9:21-44.

41. Li H, Qu JP, Li T, Wirth S, Zhang Y, Zhao X, et al. Diet simplification selects for high gut microbial diversity and strong fermenting ability in high-altitude pikas. Appl Microbiol Biotechnol. 2018;102:6739-51.

42. Lozupone CA, Stombaugh JI, Gordon JI, Jansson JK, Knight R. Diversity, stability and resilience of the human gut microbiota. Nature. 2012;489:220-30.

43. Ley RE, Peterson DA, Gordon JI. Ecological and evolutionary forces shaping microbial diversity in the human intestine. Cell. 2006; 124:837-48.

44. Bekele AZ, Koike S, Kobayashi Y. Phylogenetic diversity and dietary association of rumen Treponema revealed using groupspecific $16 \mathrm{~S}$ rRNA gene-based analysis. FEMS Microbiol Lett. 2011;316:51-60.

45. Dai X, Tian Y, Li J, Su X, Wang X, Zhao S, et al. Metatranscriptomic analyses of plant cell wall polysaccharide degradation by microorganisms in cow rumen. Appl Environ Microbiol. 2014;81:1375-86.

46. Mackie RI, Aminov RI, Hu W, Klieve AV, Ouwerkerk D, Sundset MA, et al. Ecology of uncultivated Oscillospira species in the rumen of cattle, sheep, and reindeer as assessed by microscopy and molecular approaches. Appl Environ Microbiol. 2003;69:6808-15.

47. Krause DO, Dalrymple BP, Smith WJ, Mackie RI, Mcsweeney CS. 16S rDNA sequencing of Ruminococcus albus and Ruminococcus flavefaciens: design of a signature probe and its application in adult sheep. Microbiology. 1999;145:1797-807.

48. Ziętak M, Kovatcheva-Datchary P, Markiewicz LH, Ståhlman M, Kozak LP, Bäckhed F. Altered microbiota contributes to reduced dietinduced obesity upon cold exposure. Cell Metab. 2016;23:1216-23.

49. Martínez I, Perdicaro DJ, Brown AW, Hammons S, Carden TJ, Carr TP, et al. Diet-Induced alterations of host cholesterol metabolism are likely to affect the gut microbiota composition in hamsters. Appl Environ Microbiol. 2013;79:516-24.

50. Worthmann A, John C, Rühlemann MC, Baguhl M, Heinsen FA, Schaltenberg N, et al. Cold-induced conversion of cholesterol to bile acids in mice shapes the gut microbiome and promotes adaptive thermogenesis. Nat Med. 2017;23:839-49.

51. Turnbaugh PJ, Ley RE, Mahowald MA, Magrini V, Mardis ER, Gordon JI. An obesity-associated gut microbiome with increased capacity for energy harvest. Nature. 2006;444:1027-31.

52. Li G, Xie C, Lu S, Nichols RG, Tian Y, Li L, et al. Intermittent fasting promotes white adipose browning and decreases obesity by shaping the gut microbiota. Cell Metab. 2017;26:672-85.
53. Royall D, Wolever TM, Jeejeebhoy KN. Clinical significance of colonic fermentation. Am J Gastroenterol. 1990;85:1307-12.

54. Byrne CS, Chambers ES, Morrison DJ, Frost G. The role of short chain fatty acids in appetite regulation and energy homeostasis. Int J Obes. 2015;39:1331-8.

55. Brown AJ, Goldsworthy SM, Barnes AA, Eilert MM, Tcheang L, Daniels D Muir AL, et al. The orphan G protein-coupled receptors GPR41 and GPR43 are activated by propionate and other short chain carboxylic acids. J Biol Chem. 2003;278:11312-9.

56. Tazoe H, Otomo Y, Kaji I, Tanaka R, Karaki S, Kuwahara A. Roles of short-chain fatty acids receptors, GPR41 and GPR43 on colonic functions. J Physiol Pharm. 2008;59:251-62.

57. Kaji I, Karaki S, Tanaka R, Kuwahara A. Density distribution of free fatty acid receptor 2 (FFA2)-expressing and GLP-1-producing enteroendocrine $\mathrm{L}$ cells in human and rat lower intestine, and increased cell numbers after ingestion of fructo-oligosaccharide. J Mol Histol. 2011;42:27-38.

58. Chambers ES, Morrison DJ, Frost G. Control of appetite and energy intake by SCFA: what are the potential underlying mechanisms? Proc Nutr Soc. 2015;74:328-36.

59. Christiansen $\mathrm{CB}$, Gabe MBN, Svendsen B, Dragsted LO, Rosenkilde MM, Holst JJ. The impact of short-chain fatty acids on GLP-1 and PYY secretion from the isolated perfused rat colon. Am J Physiol Gastrointest Liver Physiol. 2018;315:G53-65.

60. Aukema HM, Davidson LA, Pence BC, Jiang YH, Lupton JR, Chapkin RS. Butyrate alters activity of specific cAMP-receptor proteins in a transgenic mouse colonic cell line. J Nutr. 1997;127:18-24.

61. Wang A, Si H, Liu D, Jiang H. Butyrate activates the cAMPprotein kinase A-cAMP response element-binding protein signaling pathway in Caco-2 cells. J Nutr. 2012;142:1-6.

62. Liu L, Wang Y, Fan Y, Li CL, Chang ZL. IFN-gamma activates cAMP/PKA/CREB signaling pathway in murine peritoneal macrophages. J Interferon Cytokine Res. 2004;24:334-42.

63. Luo X, Jia R, Zhang Q, Sun B, Yan J. Cold-induced browning dynamically alters the expression profiles of inflammatory adipokines with tissue specificity in mice. Int $\mathrm{J}$ Mol Sci. 2016;17:795.

64. Dong M, Yang X, Lim S, Cao Z, Honek J, Lu H, et al. Cold exposure promotes atherosclerotic plaque growth and instability via UCP1-dependent lipolysis. Cell Metab. 2013;18:118-29.

65. Raybould HE. Gut chemosensing: interactions between gut endocrine cells and visceral afferents. Auton Neurosci. 2010; 153:41-46

66. Lopes JG, Sourjik V. Chemotaxis of Escherichia coli to major hormones and polyamines present in human gut. ISME J. 2018;12:2736-47.

67. Reigstad CS, Salmonson CE, Rainey JF, Szurszewski JH, Linden DR, Sonnenburg JL, et al. Gut microbes promote colonic serotonin production through an effect of short-chain fatty acids on enterochromaffin cells. FASEB J. 2015;29:1395-403.

68. Cogan TA, Thomas AO, Rees LE, Taylor AH, Jepson MA, Williams $\mathrm{PH}$, et al. Norepinephrine increases the pathogenic potential of Campylobacter jejuni. Gut. 2007;56:1060-5.

69. Zhang XY, Zhang Q, Wang DH. Pre- and post-weaning cold exposure does not lead to an obese phenotype in adult Brandt's voles (Lasiopodomys brandtii). Horm Behav. 2011;60:210-8.

70. Zhao ZJ, Wang DH. Effects of diet quality on energy budgets and thermogenesis in Brandt's voles. Comp Biochem Physiol A Mol Integr Physiol. 2007;148:168-77.

71. Zhang XY, Lou MF, Shen W, Fu RS, Wang DH. A maternal lowfiber diet predisposes offspring to improved metabolic phenotypes in adulthood in an herbivorous rodent. Physiol Biochem Zool. 2017;90:75-84. 